\title{
Sensitivity of the Cherenkov Telescope Array to emission from the gamma-ray counterparts of neutrino events
}

\author{
Olga Sergijenko, ${ }^{a, *}$ Anthony M. Brown, ${ }^{b}$ Damiano Fiorillo, ${ }^{c}$ Alberto Rosales de \\ León, ${ }^{b}$ Konstancja Satalecka, ${ }^{d}$ Chun Fai Tung, ${ }^{e}$ René Reimann, ${ }^{f}$ Theo Glauch $^{g}$ and \\ Ignacio Taboada ${ }^{e}$ on behalf of the CTA Consortium and the FIRESONG Team \\ Collaboration \\ (a complete list of authors can be found at the end of the proceedings) \\ ${ }^{a}$ Astronomical Observatory, Taras Shevchenko National University of Kyiv, Kyiv, Ukraine \\ ${ }^{b}$ Centre for Advanced Instrumentation (CfAI), Department of Physics, University of Durham, Durham, UK \\ ${ }^{c}$ Universita degli Studi di Napoli “Federico II” and INFN - Sezione di Napoli, Napoli, Italy \\ ${ }^{d}$ DESY, 15738 Zeuthen, Germany \\ ${ }^{e}$ Center for Relativistic Astrophysics and School of Physics, Georgia Institute of Technology, Atlanta, GA \\ 30332, USA \\ ${ }^{f}$ Institut of Physics, Johannes Gutenberg University Mainz, 55128 Mainz, Germany \\ ${ }^{g}$ Technische Universität München, Physik-Department, D-85748 Garching bei München, Germany \\ E-mail: olga.sergijenko.astro@gmail.com, anthony.brown@durham.ac.uk, \\ damianofg@gmail.com, alberto.rosales-de-leon@durham.ac.uk, \\ konstancja.satalecka@desy.de, ctung6@gatech.edu, nerreimann@uni-mainz.de, \\ theo.glauch@tum.de, itaboada@gatech.edu
}

We investigate the possibility of detection of the VHE gamma-ray counterparts to the neutrino astrophysical sources within the Neutrino Target of Opportunity (NToO) program of CTA using the populations simulated by the FIRESONG software to resemble the diffuse astrophysical neutrino flux measured by IceCube. We derive the detection probability for different zenith angles and geomagnetic field configurations. The difference in detectability of sources between CTA-North and CTA-South for the average geomagnetic field is not substantial. We investigate the effect of a higher night-sky background and the preliminary CTA Alpha layout on the detection probability.

$37^{\text {th }}$ International Cosmic Ray Conference (ICRC 2021)

July 12th-23rd, 2021

Online - Berlin, Germany

\footnotetext{
*Presenter
} 


\section{Introduction}

The Cherenkov Telescope Array will be the next generation ground-based imaging atmospheric Cherenkov telescope (IACT) array. Building upon the strengths of the current generation of telescopes, such as H.E.S.S., MAGIC and VERITAS, CTA will detect gamma rays from the energy of $20 \mathrm{GeV}$ to above $300 \mathrm{TeV}$, with an order of magnitude improvement in sensitivity, as well as improved angular and energy resolution compared to the current generation IACTs. To achieve this improved performance CTA will consist of three telescope sizes, small, medium and large (SST, MST and LST respectively), with each one optimised for a specific energy range. CTA will consist of two arrays, one in each hemisphere, with the northern array (CTA-N) consisting of 13 telescopes spread over $0.5 \mathrm{~km}^{2}$ in La Palma, Spain, and the southern array (CTA-S) consisting of 51 telescopes spread over $4 \mathrm{~km}^{2}$ near Paranal, Chile.

In the context of the origin of transient astrophysical messengers, like neutrinos, CTA has several important performance characteristics. Firstly, CTA telescopes are designed to rapidly reposition to any location in the sky. As a point of reference, the LSTs can re-position to anywhere in the sky above $30^{\circ}$ in just 20 seconds, thus minimising the time between receiving alerts from other observatories, such as IceCube, and starting observations. Secondly, the field-of-view (FoV) for LSTs will be $\sim 4^{\circ}$, for MSTs $\sim 8^{\circ}$ and for SSTs $\sim 10^{\circ}$.

Astrophysical sources capable of hadronic acceleration to relativistic energies have long been believed to be sources of astrophysical neutrinos, with the neutrinos originating from the decay of charged mesons created by the relativistic hadrons interacting with ambient gas and/or ambient radiation within the astrophysical source. The decay of these mesons results in the emission of neutrinos and gamma rays. As such, assuming that the photon opacity of the emission region is low enough to allow gamma rays to escape, searching for spatially and temporally correlated gamma-ray and neutrino emission allows us to constrain the origin of the astrophysical neutrinos observed by the IceCube Neutrino Observatory.

In April 2016, IceCube initiated a Realtime alert program in which neutrinos with high probability of being of astrophysical origin are reported in realtime via the Gamma-ray-burst Coordinate Network (GCN) [1]. The program reports $v_{\mu}$ candidate events, which have the advantage of well-localized angular positions, typically $\Delta \psi \sim 0.5^{\circ}$. Starting on June 17, 2019, a new version of the realtime program became operational [2]. In this updated system, two streams of neutrinos are provided. The Gold stream delivers $\sim 10$ events per year with an average astrophysical signal purity of 50\%. The Bronze stream has an average purity of $30 \%$ for an additional $\sim 20$ alerts per year.

In the Key Science Project proposed in the CTA Science Book [3] a total of 5 hours per site, per year, has been allocated to high-energy neutrino events observations, during the first 10 years of CTA operation. In these proceedings, we outline the ongoing work to quantify the efficiency with which CTA will observe gamma-ray emission from IceCube 'Gold' $v_{\mu}$ alerts and all-sky 'hot-spots' (i.e. highest significance positions). The structure of the proceedings is as follows: in Section 2 we outline the simulation tool set we used to simulate IceCube alerts, in Section 3 we outline our conversion from the neutrino flux to the expected gamma-ray flux. Section 4 describes our simulations of CTA response to the expected gamma-ray flux, while in Section 5 we state our results. 


\section{FIRESONG}

The sources of astrophysical high-energy neutrinos have not been unequivocally identified. Observations of TXS 0506+056 provide evidence that blazars or a sub-population of blazars are possibly responsible for a fraction of the neutrino flux [4, 5]. Nevertheless, evidence has only been claimed for one blazar/neutrino correlation therefore one has to be careful while extending its properties to describe the whole population of neutrino sources. The population of potential neutrino sources responsible for this flux can be parametrized in terms of local density (local density rate) vs. neutrino luminosity (flare energy) for steady (flaring) sources [6].

FIRESONG [7] is an open-source software that simulates neutrino source populations for a given local density and neutrino luminosity, by making additional assumptions on the source density evolution and their luminosity functions. In these proceedings we explore a source population which does not evolve with redshift (which is a simplified way to describe blazars) and one that follows the star formation rate (SFR) evolution. In both populations, sources are described as standard candles, i.e., all sources share the same neutrino luminosity. We also assume that the specific simulated class is responsible for $100 \%$ of the astrophysical neutrino flux [8]. FIRESONG is able to describe alerts with the same characteristics as those reported by IceCube. It should be noted that the rate of alert and its declination dependence depend on the IceCube response, notably the effective area. However, the redshift distribution of the sources of alerts depends on the cosmology model and the properties of the assumed population. For this work we have used the $\Lambda \mathrm{CDM}$ cosmological model with the parameters derived from the Planck-2015 data [9]: $\Omega_{M}=0.308, \Omega_{\Lambda}=0.692$ and $h=0.678$.

\subsection{TXS-like flaring sources}

The first simulated scenario is modeled after the neutrino flare of TXS 0506+056 (TXS) in the season 2014-2015. We follow the model suggested by [10] in which only a fraction of blazars, including TXS, are responsible for the astrophysical neutrino flux. We will call this population TXS-like sources. The local burst density rate can be related to the local density of blazars as:

$$
\dot{\rho}=F \times \rho_{\mathrm{BL}} .
$$

where $F$ is a fraction of the total number of blazars. As described before, we assume these sources are neutrino standard candles and display no evolution in the density rate. We also assume all TXS-like sources have the same flare duration in their local reference frame. Correcting the 110 days flare of TXS [4] for redshift, this corresponds to 82 days. Saturating the astrophysical neutrino flux with these flares results in a uniquely defined burst energy as a function of $F$.

\subsection{Steady sources}

The second simulated scenario is that the diffuse neutrino flux is due to steady neutrino sources. Although IceCube has not yet resolved a point source above $5 \sigma$ significance, there are sources that have exceeded the IceCube sensitivity (Note that sensitivity in IceCube has a different definition than for IACTs; see Ref. [11]). These sources will be of interest for the NToO program of CTA. Assuming the neutrino sources are i) standard candles, ii) follow the star formation evolution from 
[12] or a flat redshift evolution, and iii) saturate the astrophysical neutrino flux [8], we can simulate the whole-sky source population with different local density conditions. Then, the flux from each neutrino source is compared with IceCube sensitivity [11]. The sources that exceed IceCube sensitivity are used as seeds for the NToO for CTA.

\section{VHE gamma-ray emission accompanying the neutrino emission}

In order to calculate the gamma-ray flux emitted together with neutrinos we assume that they are produced in proton interactions with the surrounding photon field ( $p \gamma$ interactions) as usually postulated for Active Galactic Nuclei (AGN), and do not consider any additional absorption or cascading of $\gamma$ rays inside the source. The secondary pions and other particles decay to neutrinos or gamma rays and in the simplest case the relation between the gamma ray and neutrino production rates is:

$$
\frac{1}{3} \sum_{\alpha} E_{\nu}^{2} A_{v_{\alpha}}\left(E_{\nu}\right)=\frac{K_{\pi}}{4} E_{\gamma}^{2} A_{\gamma}\left(E_{\gamma}\right)
$$

where $\mathrm{E}_{\gamma}=2 \mathrm{E}_{v}$ and $\mathrm{K}_{\pi}=1$ is a factor which accounts for the ratio of charged to neutral pions for $p \gamma$ interactions (for full derivation see e.g. [13]).

In the case of TXS-like sources we adapt the phenomenological model of [10]. The emerging gamma-ray flux is given by:

$$
\frac{d N_{\gamma}}{d E}=A_{\nu} E^{-2} e^{\left(-E_{L} / E-E / E_{H}\right)}
$$

where $\mathrm{E}_{L}$ and $\mathrm{E}_{H}$ are low- and high-energy cutoffs, and $\mathrm{A}_{v}$ is proportional to the simulated neutrino flux normalization. In a case of TXS 0506+056 located at redshift $z=0.335$, the $\mathrm{E}_{L}=0.1 \mathrm{TeV}$ and $\mathrm{E}_{H}=20 \mathrm{TeV}$ (see [10]). For the sources located at different redshifts, we scale those values accordingly.

\section{CTA simulations}

To simulate the CTA follow-up observations of the neutrino alerts we use the ctools package with gammalib [14]. We employed the prod3b-v2 CTA instrument response functions (IRFs) for the standard IRF set for the preliminary Omega Configuration, and the prod3b CTA IRFs for the preliminary Alpha Configuration sub-array and high night sky background (NSB) conditions. When possible, 1,000 FIRESONG alerts were considered for each of our density-luminosity sample using the different IRFs configurations. We obtain the redshift, spectrum normalization and declination for the alerts from running FIRESONG, while the right ascension is assigned randomly. For all sources, we take into account extragalactic background light (EBL) absorption [15].

The first set of IRFs considered corresponds to the preliminary North and South Omega configuration: 4 LSTs and 15 MSTs for CTA-N and 4 LSTs, 25 MSTs and 70 SSTs for CTA-S [16]. The Omega IRFs set contains three zenith angle observation options at $20^{\circ}, 40^{\circ}$ and $60^{\circ}$; and it also accounts for the azimuth dependence coming from the magnetic field pointing direction: North, South or an average over the azimuth direction. The second IRFs set is the preliminary Alpha Configuration array, which was expected during the construction phase of CTA. A sub-array with a lower number of operational telescopes: 15 MSTs and 50 SSTs for CTA-S and 4 LSTs and 5 MSTs 

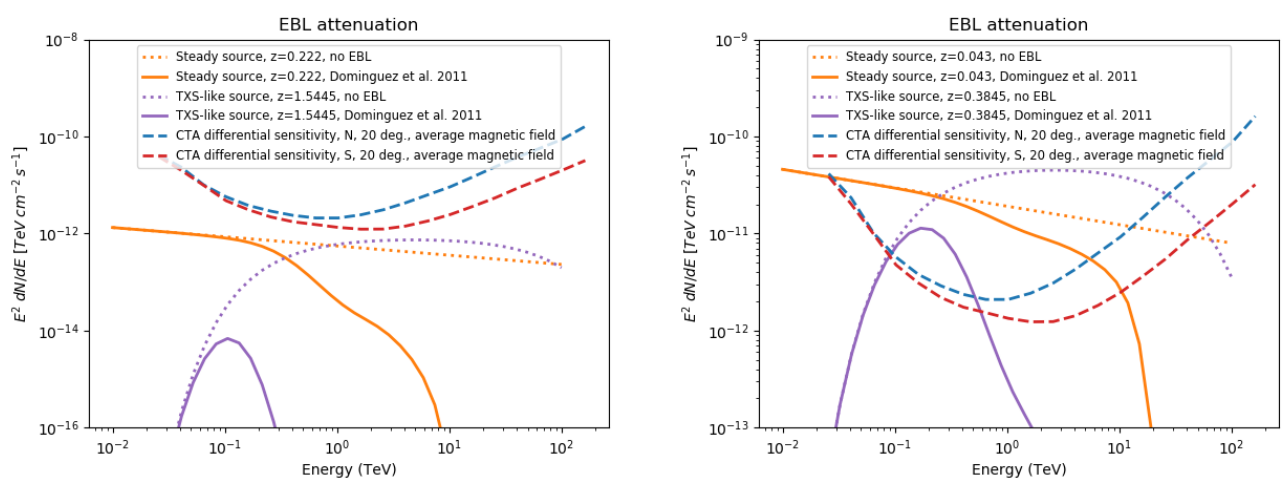

Figure 1: The energy spectra without and with the EBL attenuation vs the CTA differential sensitivity for the undetected (left) and detected (right) sources.

for CTA-N. Note that it differs from the current official Alpha Configuration which includes 4 LSTs + 9 MSTs for CTA-N and 14 MSTs + 37 SSTs for CTA-S.

For each alert in the Omega Configuration, we simulate the photon events list for $30 \mathrm{~min}$ of either North or South site observations with a $5.0^{\circ}$ ROI centered at a source (with the tool ctobsssim). We consider the following energy ranges: $0.03-200 \mathrm{TeV}$ for $20^{\circ}, 0.04-200 \mathrm{TeV}$ for $40^{\circ}$ and $0.110-200 \mathrm{TeV}$ for $60^{\circ}$ zenith angles (the lower and upper limits are defined by the IRFs). The energy dispersion effect was also considered in the simulated observations. We then perform a maximum likelihood fitting using the tool ctlike in an unbinned mode. The test statistic (TS) equal or higher than 25 qualifies as a source detection at the $\sim 5 \sigma$ level. For the Alpha configuration, zenith angle and azimuth pointing direction combinations were the same as in the Omega Configuration. ctools simulations for SFR and flat evolution steady sources followed the same specifications (ROI, observation times, zenith angles).

The average Light of the Night Sky (LoNS) on La Palma site is around $1.7 \times 10^{12} \mathrm{ph} \mathrm{m}^{-2} \mathrm{sr}^{-1}$ $\mathrm{s}^{-1}$ on the IACTs sensitive range [17]. Measurements on both sites (CTA-N and CTA-S) showed similar NSB levels [18]. CTA observations up to 5 times the NSB level found in dark sky patches away from the Galactic Plane are anticipated when the moon is above the horizon. Simulations with our last set of IRFs tested this scenario, only 4 selected cases were at our disposal within the prod3b IRFs version: CTA-N $20^{\circ}$ for North, South and average pointing direction in azimuth, and CTA-S $20^{\circ}$ average in azimuth.

The ctools simulations for the TXS-like flaring sources followed the same scheme as in the steady source scenario. The previously described 3 IRFs sets were used. The IRF specifications such as energy range, ROI, zenith angles and magnetic field azimuth dependence were the same as in the steady source scenario.

\section{Results}

TXS-like flaring blazars. Figure 2 shows the detection probability as a function of flaring sources fraction $F$ for 30 min observation time for CTA-N and CTA-S, assuming different 

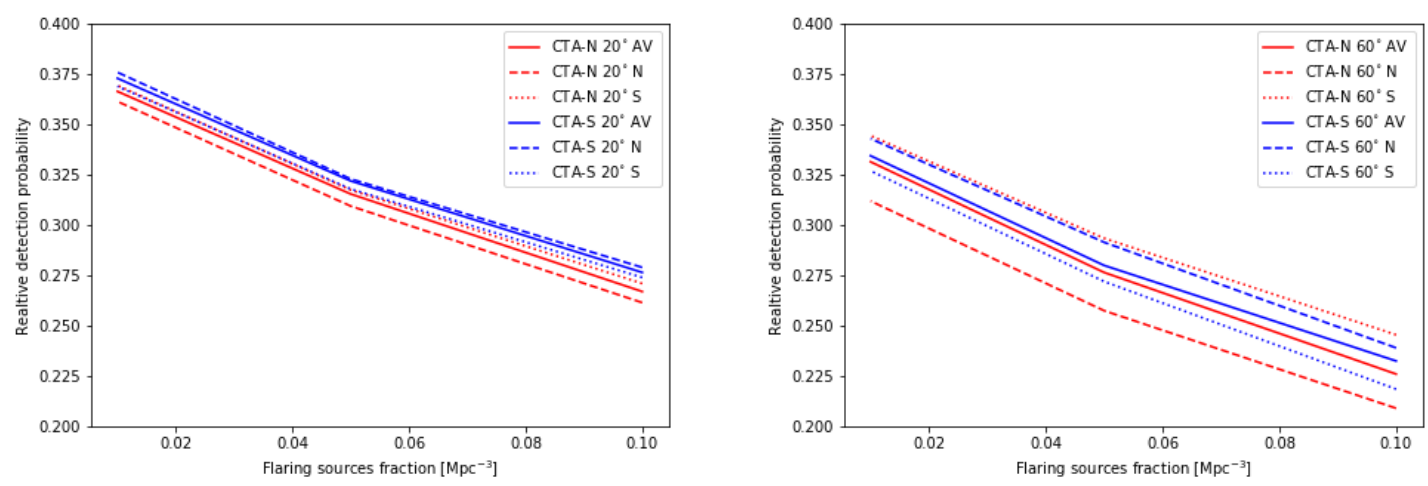

Figure 2: Detection probability as a function of flaring sources fraction $F$ for $30 \mathrm{~min}$ observation time, assuming CTA-N (red) and CTA-S (blue) observations with zenith angle of $20^{\circ}$, and $60^{\circ}$. The solid, dashed and dotted lines represent 3 azimuth angle alignments: average, North and South.

zenith and azimuth angle configurations. This probability, taking into account a background event contamination of $50 \%$, is highest for the population with $1 \%$ of flaring sources.

In this scenario, the detection probability is almost identical for CTA-N and CTA-S (difference $<1 \%$ ) with average azimuth direction. There's also almost no difference for $20^{\circ}$ and $40^{\circ}$ zenith IRFs (again $<1 \%$ ), but with higher zenith angles $\left(60^{\circ}\right)$ we measure a decrease of $\sim 4 \%$. The influence of magnetic field is minimal, although slightly more evident for CTA-N and for high zenith angles.

Steady sources. For the steady sources case we present the detection probability as a function of source luminosity $(L)$ and local density $(\rho)$. Fig. 3 shows our results for sources following the SFR evolution for 30 min observations with CTA-N and CTA-S respectively. The plots in rows from top to bottom represent results for zenith angle of $20^{\circ}, 40^{\circ}$ and $60^{\circ}$, while the columns separate three different azimuth angle alignments: North, average and South (from left to right).

With low to mid zenith angle observations $\left(20^{\circ}-40^{\circ}\right)$ CTA-N will be able to detect all sources down to the density of $\rho=10^{-9} \mathrm{Mpc}^{-3}$. A drastic performance loss, up to $65 \%$, is measured at high zeniths $\left(60^{\circ}\right)$. The effect of the geomagnetic field is reflected in the 10-30\% difference in detection probability for North/South azimuth directions for low to high zeniths. The CTA-S array shows a similar response as CTA-N (within $10 \%$ for average azimuth). The main differences are a higher performance loss, up to $70 \%$, at high zeniths $\left(60^{\circ}\right)$ and a smaller influence of geomagnetic field (5-15\% difference in detection probability for North/South azimuth directions for low to high zeniths).

The higher NSB levels do not influence the detection probability for sources with $\rho>10^{-10}$ $\mathrm{Mpc}^{-3}$ and $L<10^{55} \mathrm{erg} / \mathrm{yr}$. For lower densities and higher luminosities the detection rate decreases by $10-15 \%$. This effect is similar for both considered arrays and cosmological evolution scenarios.

In the Alpha configuration used in this study the CTA-N performance is almost the same as for the full Omega array. On the other hand, the lack of LSTs in the Alpha configuration of the CTA-S drastically diminishes its detection capability. The effect is most visible for $\rho<10^{-9}$ and $L>10^{55}$ erg/yr. In the most extreme cases the detection probability drops by one third for zenith angles of $20^{\circ}$, for $40^{\circ}$ by half, and with $60^{\circ}$ at most $10 \%$ of sources are detected.

We define the CTA redshift reach as the maximum redshift for $90 \%$ of the detected sources 


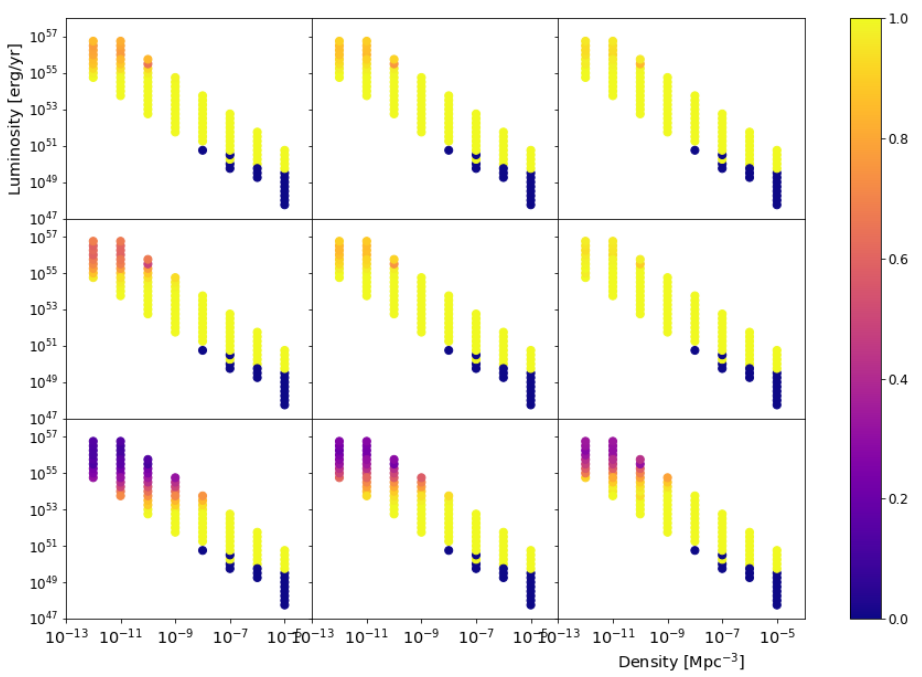

Figure 3: Detection probability (color scale) as a function of source luminosity $(L)$ and local density $(\rho)$ for sources following the SFR redshift evolution for $30 \mathrm{~min}$ observations with CTA-N. The plots in rows from top to bottom represent results for zenith angles of $20^{\circ}, 40^{\circ}$ and $60^{\circ}$, while the columns separate 3 azimuth angle alignments: North, average and South (from left to right). An abrupt drop in the detection probability from 1 to 0 occurs for $L$ and $\rho$ for which the FIRESONG output contained no alerts.

in the distribution for each local density (the last decile was cut due to the low number of detected sources). Figure 4 shows an example plot for the steady SFR evolution case at $\rho=10^{-12} \mathrm{Mpc}^{-3}$ observed with CTA-N and CTA-S. The highest redshift reach among our simulations is given by this density and is up to $z \sim 2.8$. For observations at $20^{\circ}$ and $40^{\circ}$ zenith angle the redshift reach is similar, however there is a big drop for $60^{\circ}$. This is a general behaviour for all the density range considered. For sources with the flat redshift evolution the trends are similar as those described above, but the redshift reach is lower than for the SFR evolution.

\section{Acknowledgments}

$\mathrm{KS}, \mathrm{AB}, \mathrm{ARdL}$ and $\mathrm{OS}$ gratefully acknowledge financial support from the agencies and organizations listed here: www.cta-observatory.org/consortium_acknowledgments. This work was performed within the CTA Transients Physics Working Group. KS acknowledge the the European Union's Horizon 2020 Programme under the support of the European Union's Horizon 2020 Programme under the AHEAD2020 project (grant agreement $n$. 871158). CFT and IT acknowledge support by NSF grant PHY-1505230. ARdL acknowledges the support of the National Council for Science and Technology from Mexico (CONACYT).

This research made use of ctools, a community-developed analysis package for Imaging Air Cherenkov Telescope data. ctools is based on GammaLib, a community-developed toolbox for the scientific analysis of astronomical gamma-ray data.

\section{References}

[1] M.G. Aarsten, et al. (IceCube Collaboration). Astropart. Phys. 92 (2017) 30 

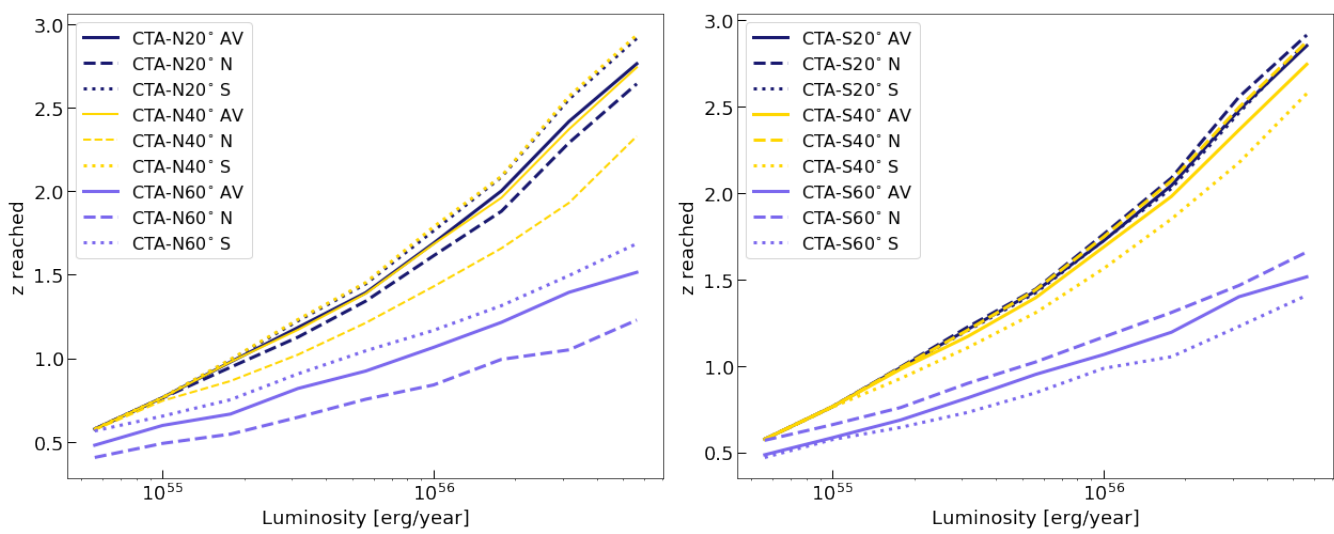

Figure 4: Redshift reach from CTA-N (left) and CTA-S (right) simulations for steady sources at density $\rho=10^{-12} \mathrm{Mpc}^{-3}$ following SFR evolution. Zenith angles of $20^{\circ}, 40^{\circ}$ and $60^{\circ}$ are considered. The solid lines represent the IRF with an average magnetic field, while the dashed (dotted) lines are for the pointing North (South) direction.

[2] E. Blauffus, T. Kinstcher, L. Lu and C.F. Tung for the IceCube collaboration. These proceedings, PoS(ICRC2019)1021.

[3] B.S. Acharya et al. [CTA Consortium], Science with the Cherenkov Telescope Array. World Scientific (2019). https://doi.org/10.1142/10986

[4] M. G. Aartsen et al. [IceCube Collaboration], Science 361, no. 6398, 147 (2018)

[5] M. G. Aartsen et al. [IceCube, Fermi-LAT, MAGIC, AGILE, ASAS-SN, HAWC, H.E.S.S., INTEGRAL, Kanata, Kiso, Kapteyn, Liverpool Telescope, Subaru, Swift NuSTAR, VERITAS, VLA/17B-403 Collaboration], Science 361, no. 6398, eaat1378 (2018)

[6] M. Kowalski. Proceedings of the Eur. Cosmic Ray Symp. 2014. arXiv:1411.4385

[7] Tung et al., Journal of Open Source Software, 6(61), 3194 (2021)

[8] M. G. Aartsen et al. [IceCube Collaboration], Proceedings of ICRC 2017. arXiv:1710.01191 [astroph.HE].

[9] P.A.R. Ade et al. [Planck Collaboration], Astron. Astrophys. 594, A13 (2016)

[10] F. Halzen, A. Kheirandish and T. Weisgarber and S.P. Wakely ApJ 874 (2019) L9

[11] M. G. Aartsen et al. [IceCube Collaboration], Eur. Phys. J. C 79, no. 3, 234 (2019)

[12] P. Madau and M. Dickinson, Ann. Rev. Astron. Astrophys. 52, 415 (2014)

[13] Ahlers M., Halzen F., 2018, PrPNP, 102, 73

[14] J. Knoedlseder et al., A \& A, 593, A1 (2016).

[15] A. Dominguez et al., Mon. Not. Roy. Astron. Soc., 410, 2556 (2011)

[16] A. Acharyya et al. [CTA Collaboration], Astroparticle Physics, 111, 35 (2019).

[17] R. Mirzoyan et al., New Astronomy Reviews, 42, no. 6, 547 (1998).

[18] T. Hassan et al., Astroparticle Physics, 93, 76 (2017). 


\section{Full Authors List: CTA Consortium and the FIRESONG Team Collaboration}

\section{The Cherenkov Telescope Array Consortium July 2021 Authors}

H. Abdalla ${ }^{1}$, H. Abe ${ }^{2}$, S. Abe ${ }^{2}$, A. Abusleme ${ }^{3}$, F. Acero ${ }^{4}$, A. Acharyya ${ }^{5}$, V. Acín Portella ${ }^{6}$, K. Ackley ${ }^{7}$, R. Adam ${ }^{8}$, C. Adams $^{9}$, S.S. Adhikari ${ }^{10}$, I. Aguado-Ruesga ${ }^{11}$, I. Agudo ${ }^{12}$, R. Aguilera ${ }^{13}$, A. Aguirre-Santaella ${ }^{14}$, F. Aharonian ${ }^{15}$, A. Alberdi ${ }^{12}$, R. Alfaro ${ }^{16}$, J. Alfaro ${ }^{3}$, C. Alispach ${ }^{17}$, R. Aloisio ${ }^{18}$, R. Alves Batista ${ }^{19}$, J.-P. Amans ${ }^{20}$, L. Amati ${ }^{21}$, E. Amato ${ }^{22}$, L. Ambrogi ${ }^{18}$, G. Ambrosi ${ }^{23}$, M. Ambrosio ${ }^{24}$, R. Ammendola ${ }^{25}$, J. Anderson ${ }^{26}$, M. Anduze ${ }^{8}$, E.O. Angüner ${ }^{27}$, L.A. Antonelli ${ }^{28}$, V. Antonuccio ${ }^{29}$, P. Antoranz ${ }^{30}$,

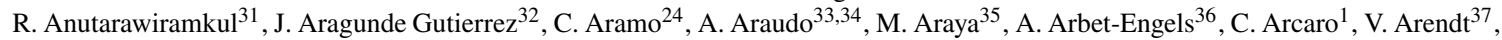
C. Armand ${ }^{38}$, T. Armstrong ${ }^{27}$, F. Arqueros ${ }^{11}$, L. Arrabito ${ }^{39}$, B. Arsioli ${ }^{40}$, M. Artero ${ }^{41}$, K. Asano ${ }^{2}$, Y. Ascasíbar ${ }^{14}$, J. Aschersleben ${ }^{42}$, M. Ashley ${ }^{43}$, P. Attinà ${ }^{44}$, P. Aubert ${ }^{45}$, C. B. Singh ${ }^{19}$, D. Baack ${ }^{46}$, A. Babic ${ }^{47}$, M. Backes ${ }^{48}$, V. Baena ${ }^{13}$, S. Bajtlik ${ }^{49}$, A. Baktash ${ }^{50}$, C. Balazs ${ }^{7}$, M. Balbo ${ }^{38}$, O. Ballester ${ }^{41}$, J. Ballet ${ }^{4}$, B. Balmaverde ${ }^{44}$, A. Bamba ${ }^{51}$, R. Bandiera ${ }^{22}$, A. Baquero Larriva ${ }^{11}$, P. Barai ${ }^{19}$, C. Barbier ${ }^{45}$, V. Barbosa Martins ${ }^{52}$, M. Barcelo ${ }^{53}$, M. Barkov ${ }^{54}$, M. Barnard ${ }^{1}$, L. Baroncelli ${ }^{21}$, U. Barres de Almeida ${ }^{40}$, J.A. Barrio ${ }^{11}$, D. Bastieri ${ }^{55}$, P.I. Batista ${ }^{52}$, I. Batkovic ${ }^{55}$, C. Bauer ${ }^{53}$, R. Bautista-González ${ }^{56}$, J. Baxter ${ }^{2}$, U. Becciani ${ }^{29}$, J. Becerra González ${ }^{32}$, Y. Becherini ${ }^{57}$, G. Beck ${ }^{58}$, J. Becker Tjus ${ }^{59}$, W. Bednarek ${ }^{60}$, A. Belfiore ${ }^{61}$, L. Bellizzi ${ }^{62}$, R. Belmont ${ }^{4}$, W. Benbow ${ }^{63}$, D. Berge ${ }^{52}$, E. Bernardini ${ }^{52}$, M.I. Bernardos ${ }^{55}$, K. Bernlöhr ${ }^{53}$, A. Berti ${ }^{64}$, M. Berton ${ }^{65}$, B. Bertucci ${ }^{23}$, V. Beshley ${ }^{66}$, N. Bhatt ${ }^{67}$, S. Bhattacharyya ${ }^{67}$, W. Bhattacharyya ${ }^{52}$, S. Bhattacharyya ${ }^{68}$, B. Bi ${ }^{69}$, G. Bicknell ${ }^{70}$, N. Biederbeck ${ }^{46}$, C. Bigongiari ${ }^{28}$, A. Biland ${ }^{36}$, R. Bird ${ }^{71}$, E. Bissaldi ${ }^{72}$, J. Biteau ${ }^{73}$, M. Bitossi ${ }^{74}$, O. Blanch ${ }^{41}$, M. Blank ${ }^{50}$, J. Blazek ${ }^{33}$, J. Bobin ${ }^{75}$, C. Boccato ${ }^{76}$, F. Bocchino ${ }^{77}$, C. Boehm ${ }^{78}$, M. Bohacova ${ }^{33}$, C. Boisson ${ }^{20}$, J. Boix ${ }^{41}$, J.-P. Bolle ${ }^{52}$, J. Bolmont ${ }^{79}$, G. Bonanno ${ }^{29}$, C. Bonavolonta ${ }^{24}$, L. Bonneau Arbeletche ${ }^{80}$, G. Bonnoli ${ }^{12}$, P. Bordas ${ }^{81}$, J. Borkowski ${ }^{49}$, S. Bórquez ${ }^{35}$, R. Bose ${ }^{82}$, D. Bose ${ }^{83}$, Z. Bosnjak ${ }^{47}$, E. Bottacini ${ }^{55}$, M. Böttcher ${ }^{1}$, M.T. Botticella ${ }^{84}$, C. Boutonnet ${ }^{85}$, F. Bouyjou ${ }^{75}$, V. Bozhilov ${ }^{86}$, E. Bozzo ${ }^{38}$, L. Brahimi ${ }^{39}$, C. Braiding ${ }^{43}$, S. Brau-Nogué ${ }^{87}$, S. Breen ${ }^{78}$, J. Bregeon ${ }^{39}$, M. Breuhaus ${ }^{53}$, A. Brill ${ }^{9}$, W. Brisken ${ }^{88}$, E. Brocato ${ }^{28}$, A.M. Brown ${ }^{5}$, K. Brügge ${ }^{46}$, P. Brun ${ }^{89}$, P. Brun ${ }^{39}$, F. Brun ${ }^{89}$, L. Brunetti ${ }^{45}$ G. Brunetti ${ }^{90}$, P. Bruno ${ }^{29}$, A. Bruno ${ }^{91}$, A. Bruzzese ${ }^{6}$, N. Bucciantini ${ }^{22}$, J. Buckley ${ }^{82}$, R. Bühler ${ }^{52}$, A. Bulgarelli ${ }^{21}$, T. Bulik ${ }^{92}$, M. Bünning ${ }^{52}$, M. Bunse ${ }^{46}$, M. Burton ${ }^{93}$, A. Burtovoi ${ }^{76}$, M. Buscemi ${ }^{94}$, S. Buschjäger ${ }^{46}$, G. Busetto ${ }^{55}$, J. Buss ${ }^{46}$, K. Byrum ${ }^{26}$, A. Caccianiga ${ }^{95}$, F. Cadoux ${ }^{17}$, A. Calanducci ${ }^{29}$, C. Calderón ${ }^{3}$, J. Calvo Tovar $^{32}$, R. Cameron ${ }^{96}$, P. Campaña ${ }^{35}$, R. Canestrari ${ }^{91}$, F. Cangemi ${ }^{79}$, B. Cantlay ${ }^{31}$, M. Capalbi ${ }^{91}$, M. Capasso ${ }^{9}$, M. Cappi ${ }^{21}$, A. Caproni ${ }^{97}$, R. Capuzzo-Dolcetta ${ }^{28}$, P. Caraveo ${ }^{61}$, V. Cárdenas ${ }^{98}$, L. Cardiel $^{41}$, M. Cardillo ${ }^{99}$, C. Carlile ${ }^{100}$, S. Caroff ${ }^{45}$, R. Carosi ${ }^{74}$, A. Carosi ${ }^{17}$, E. Carquín ${ }^{35}$, M. Carrère ${ }^{39}$, J.-M. Casandjian ${ }^{4}$, S. Casanova ${ }^{101,53}$, E. Cascone ${ }^{84}$, F. Cassol ${ }^{27}$, A.J. Castro-Tirado ${ }^{12}$, F. Catalani ${ }^{102}$, O. Catalano ${ }^{91}$, D. Cauz ${ }^{103}$, A. Ceccanti ${ }^{64}$, C. Celestino Silva ${ }^{80}$, S. Celli ${ }^{18}$, K. Cerny ${ }^{104}$, M. Cerruti ${ }^{85}$, E. Chabanne ${ }^{45}$, P. Chadwick ${ }^{5}$, Y. Chai ${ }^{105}$, P. Chambery ${ }^{106}$, C. Champion ${ }^{85}$, S. Chandra ${ }^{1}$, S. Chaty ${ }^{4}$, A. Chen ${ }^{58}$, K. Cheng ${ }^{2}$, M. Chernyakova ${ }^{107}$, G. Chiaro ${ }^{61}$, A. Chiavassa ${ }^{64,108}$, M. Chikawa ${ }^{2}$, V.R. Chitnis ${ }^{109}$, J. Chudoba ${ }^{33}$, L. Chytka ${ }^{104}$, S. Cikota ${ }^{47}$, A. Circiello ${ }^{24,110}$, P. Clark ${ }^{5}$, M. Çolak ${ }^{41}$, E. Colombo ${ }^{32}$, J. Colome ${ }^{13}$, S. Colonges ${ }^{85}$, A. Comastri ${ }^{21}$, A. Compagnino ${ }^{91}$, V. Conforti ${ }^{21}$, E. Congiu ${ }^{95}$, R. Coniglione ${ }^{94}$, J. Conrad $^{111}$, F. Conte ${ }^{53}$, J.L. Contreras ${ }^{11}$, P. Coppi ${ }^{112}$, R. Cornat ${ }^{8}$, J. Coronado-Blazquez ${ }^{14}$, J. Cortina ${ }^{113}$, A. Costa $^{29}$, H. Costantini ${ }^{27}$, G. Cotter ${ }^{114}$, B. Courty ${ }^{85}$, S. Covino ${ }^{95}$, S. Crestan ${ }^{61}$, P. Cristofari ${ }^{20}$, R. Crocker ${ }^{70}$, J. Croston ${ }^{115}$, K. Cubuk ${ }^{93}$, O. Cuevas ${ }^{98}$, X. Cui ${ }^{2}$, G. Cusumano $^{91}$, S. Cutini2 ${ }^{23}$, A. D’Ai ${ }^{91}$, G. D'Amico ${ }^{116}$, F. D’Ammando ${ }^{90}$, P. D’Avanzo ${ }^{95}$, P. Da Vela ${ }^{74}$, M. Dadina ${ }^{21}$, S. Dai ${ }^{117}$, M. Dalchenko ${ }^{17}$, M. Dall' Ora ${ }^{84}$, M.K. Daniel ${ }^{63}$, J. Dauguet ${ }^{85}$, I. Davids ${ }^{48}$, J. Davies ${ }^{114}$, B. Dawson ${ }^{118}$, A. De Angelis ${ }^{55}$, A.E. de Araújo Carvalho ${ }^{40}$, M. de Bony de Lavergne ${ }^{45}$, V. De Caprio ${ }^{84}$, G. De Cesare ${ }^{21}$, F. De Frondat ${ }^{20}$, E.M. de Gouveia Dal Pino ${ }^{19}$, I. de la Calle ${ }^{11}$, B. De Lotto ${ }^{103}$, A. De Luca ${ }^{61}$, D. De Martino ${ }^{84}$, R.M. de Menezes $^{19}$, M. de Naurois ${ }^{8}$, E. de Oña Wilhelmi ${ }^{13}$, F. De Palma ${ }^{64}$, F. De Persio ${ }^{119}$, N. de Simone ${ }^{52}$, V. de Souza ${ }^{80}$, M. Del Santo ${ }^{91}$, M.V. del Valle ${ }^{19}$, E. Delagnes ${ }^{75}$, G. Deleglise ${ }^{45}$, M. Delfino Reznicek ${ }^{6}$, C. Delgado ${ }^{113}$, A.G. Delgado Giler ${ }^{80}$, J. Delgado Mengual ${ }^{6}$, R. Della Ceca ${ }^{95}$, M. Della Valle ${ }^{84}$, D. della Volpe ${ }^{17}$, D. Depaoli ${ }^{64,108}$, D. Depouez ${ }^{27}$, J. Devin ${ }^{85}$, T. Di Girolamo ${ }^{24,110}$, C. Di Giulio ${ }^{25}$, A. Di Piano ${ }^{21}$, F. Di Pierro ${ }^{64}$, L. Di Venere ${ }^{120}$, C. Díaz ${ }^{113}$, C. Díaz-Bahamondes ${ }^{3}$, C. Dib ${ }^{35}$, S. Diebold ${ }^{69}$, S. Digel ${ }^{96}$, R. Dima ${ }^{55}$, A. Djannati-Atai ${ }^{85}$, J. Djuvsland ${ }^{116}$, A. Dmytriiev ${ }^{20}$, K. Docher ${ }^{9}$, A. Domínguez ${ }^{11}$, D. Dominis Prester ${ }^{121}$, A. Donath ${ }^{53}$, A. Donini ${ }^{41}$, D. Dorner ${ }^{122}$, M. Doro ${ }^{55}$, R.d.C. dos Anjos ${ }^{123}$, J.-L. Dournaux ${ }^{20}$, T. Downes ${ }^{107}$, G. Drake ${ }^{26}$, H. Drass ${ }^{3}$, D. Dravins ${ }^{100}$, C. Duangchan ${ }^{31}$,

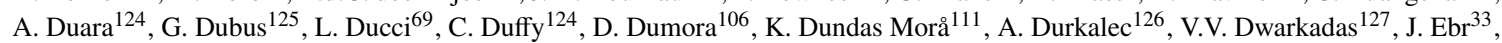
C. Eckner ${ }^{45}$, J. Eder ${ }^{105}$, A. Ederoclite ${ }^{19}$, E. Edy ${ }^{8}$, K. Egberts ${ }^{128}$, S. Einecke ${ }^{118}$, J. Eisch ${ }^{129}$, C. Eleftheriadis ${ }^{130}$, D. Elsässer ${ }^{46}$, G. Emery ${ }^{17}$, D. Emmanoulopoulos ${ }^{115}$, J.-P. Ernenwein ${ }^{27}$, M. Errando ${ }^{82}$, P. Escarate ${ }^{35}$, J. Escudero ${ }^{12}$, C. Espinoza ${ }^{3}$, S. Ettori ${ }^{21}$, A. Eungwanichayapant ${ }^{31}$, P. Evans ${ }^{124}$, C. Evoli ${ }^{18}$, M. Fairbairn ${ }^{131}$, D. Falceta-Goncalves ${ }^{132}$, A. Falcone ${ }^{133}$, V. Fallah Ramazani ${ }^{65}$, R. Falomo ${ }^{76}$, K. Farakos ${ }^{134}$, G. Fasola ${ }^{20}$, A. Fattorini ${ }^{46}$, Y. Favre ${ }^{17}$, R. Fedora ${ }^{135}$, E. Fedorova ${ }^{136}$, S. Fegan ${ }^{8}$, K. Feijen $^{118}$, Q. Feng ${ }^{9}$, G. Ferrand ${ }^{54}$, G. Ferrara ${ }^{94}$, O. Ferreira ${ }^{8}$, M. Fesquet ${ }^{75}$, E. Fiandrini ${ }^{23}$, A. Fiasson ${ }^{45}$, M. Filipovic ${ }^{117}$, D. Fink ${ }^{105}$, J.P. Finley ${ }^{137}$, V. Fioretti ${ }^{21}$, D.F.G. Fiorillo ${ }^{24,110}$, M. Fiorini ${ }^{61}$, S. Flis ${ }^{52}$, H. Flores ${ }^{20}$, L. Foffano ${ }^{17}$, C. Föhr ${ }^{53}$, M.V. Fonseca ${ }^{11}$, L. Font ${ }^{138}$, G. Fontaine ${ }^{8}$, O. Fornieri ${ }^{52}$, P. Fortin ${ }^{63}$, L. Fortson ${ }^{88}$, N. Fouque ${ }^{45}$, A. Fournier ${ }^{106}$, B. Fraga ${ }^{40}$, A. Franceschini ${ }^{76}$, F.J. Franco ${ }^{30}$, A. Franco Ordovas $^{32}$, L. Freixas Coromina ${ }^{113}$, L. Fresnillo ${ }^{30}$, C. Fruck ${ }^{105}$, D. Fugazza ${ }^{95}$, Y. Fujikawa ${ }^{139}$, Y. Fujita ${ }^{2}$, S. Fukami ${ }^{2}$, Y. Fukazawa ${ }^{140}$, Y. Fukui ${ }^{141}$, D. Fulla ${ }^{52}$, S. Funk ${ }^{142}$, A. Furniss ${ }^{143}$, O. Gabella ${ }^{39}$, S. Gabici ${ }^{85}$, D. Gaggero ${ }^{14}$, G. Galanti ${ }^{61}$, G. Galaz $^{3}$, P. Galdemard ${ }^{144}$, Y. Gallant ${ }^{39}$, D. Galloway ${ }^{7}$, S. Gallozzi ${ }^{28}$, V. Gammaldi ${ }^{14}$, R. Garcia ${ }^{41}$, E. Garcia ${ }^{45}$, E. García ${ }^{13}$, R. Garcia López ${ }^{32}$, M. Garczarczyk ${ }^{52}$, F. Gargano ${ }^{120}$, C. Gargano ${ }^{91}$, S. Garozzo ${ }^{29}$, D. Gascon ${ }^{81}$, T. Gasparetto ${ }^{145}$, D. Gasparrini2 ${ }^{25}$, H. Gasparyan ${ }^{52}$, M. Gaug ${ }^{138}$, N. Geffroy ${ }^{45}$, A. Gent ${ }^{146}$, S. Germani ${ }^{76}$, L. Gesa $^{13}$, A. Ghalumyan ${ }^{147}$, A. Ghedina ${ }^{148}$, G. Ghirlanda ${ }^{95}$, F. Gianotti ${ }^{21}$, S. Giarrusso ${ }^{91}$, M. Giarrusso ${ }^{44}$, G. Giavitto ${ }^{52}$, B. Giebels ${ }^{8}$, N. Giglietto ${ }^{72}$, V. Gika ${ }^{134}$, F. Gillardo ${ }^{45}$, R. Gimenes ${ }^{19}$, F. Giordano ${ }^{149}$, G. Giovannini ${ }^{90}$, E. Giro ${ }^{76}$, M. Giroletti ${ }^{90}$, A. Giuliani ${ }^{61}$, L. Giunti ${ }^{85}$, M. Gjaja ${ }^{9}$, J.-F. Glicenstein ${ }^{89}$, P. Gliwny ${ }^{60}$, N. Godinovic ${ }^{150}$, H. Göksu ${ }^{53}$, P. Goldoni ${ }^{85}$, J.L. Gómez ${ }^{12}$, G. Gómez-Vargas ${ }^{3}$, M.M. González ${ }^{16}$, J.M. González ${ }^{151}$, K.S. Gothe ${ }^{109}$, D. Götz ${ }^{4}$, J. Goulart Coelho $^{123}$, K. Gourgouliatos ${ }^{5}$, T. Grabarczyk ${ }^{152}$, R. Graciani ${ }^{81}$, P. Grandi ${ }^{21}$, G. Grasseau $^{8}$, D. Grasso ${ }^{74}$, A.J. Green ${ }^{78}$, D. Green ${ }^{105}$, J. Green ${ }^{28}$, T. Greenshaw ${ }^{153}$, I. Grenier ${ }^{4}$, P. Grespan ${ }^{55}$, A. Grillo ${ }^{29}$, M.-H. Grondin ${ }^{106}$, J. Grube ${ }^{131}$, V. Guarino ${ }^{26}$, B. Guest ${ }^{37}$, O. Gueta ${ }^{52}$, M. Gündüz ${ }^{59}$, S. Gunji ${ }^{154}$, A. Gusdorf ${ }^{20}$, G. Gyuk ${ }^{155}$, J. Hackfeld ${ }^{59}$, D. Hadasch ${ }^{2}$, J. Haga ${ }^{139}$, L. Hagge ${ }^{52}$, A. Hahn ${ }^{105}$, 


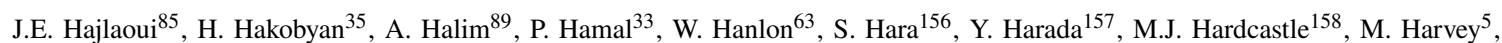
K. Hashiyama ${ }^{2}$, T. Hassan Collado ${ }^{113}$, T. Haubold ${ }^{105}$, A. Haupt ${ }^{52}$, U.A. Hautmann ${ }^{159}$, M. Havelka ${ }^{33}$, K. Hayashi ${ }^{141}$, K. Hayashi ${ }^{160}$, M. Hayashida ${ }^{161}, \mathrm{H} . \mathrm{He}^{54}$, L. Heckmann ${ }^{105}$, M. Heller ${ }^{17}$, J.C. Helo ${ }^{35}$, F. Henault ${ }^{125}$, G. Henri ${ }^{125}$, G. Hermann ${ }^{53}$, R. Hermel ${ }^{45}$, S. Hernández Cadena ${ }^{16}$, J. Herrera Llorente ${ }^{32}$, A. Herrero ${ }^{32}$, O. Hervet ${ }^{143}$, J. Hinton ${ }^{53}$, A. Hiramatsu ${ }^{157}$, N. Hiroshima ${ }^{54}$, K. Hirotani ${ }^{2}$, B. Hnatyk ${ }^{136}$, R. Hnatyk ${ }^{136}$, J.K. Hoang ${ }^{11}$, D. Hoffmann ${ }^{27}$, W. Hofmann ${ }^{53}$, C. Hoischen ${ }^{128}$, J. Holder ${ }^{162}$, M. Holler ${ }^{163}$, B. Hona ${ }^{164}$,

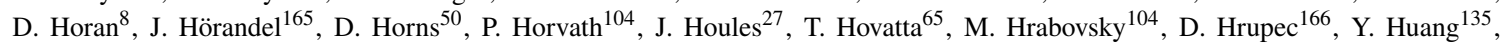
J.-M. Huet ${ }^{20}$, G. Hughes ${ }^{159}$, D. Hui ${ }^{2}$, G. Hull ${ }^{73}$, T.B. Humensky ${ }^{9}$, M. Hütten ${ }^{105}$, R. Iaria ${ }^{77}$, M. Iarlori ${ }^{18}$, J.M. Illa ${ }^{41}$, R. Imazawa ${ }^{140}$, D. Impiombato ${ }^{91}$, T. Inada ${ }^{2}$, F. Incardona ${ }^{29}$, A. Ingallinera ${ }^{29}$, Y. Inome ${ }^{2}$, S. Inoue ${ }^{54}$, T. Inoue ${ }^{141}$, Y. Inoue ${ }^{167}$, A. Insolia ${ }^{120,94}$,

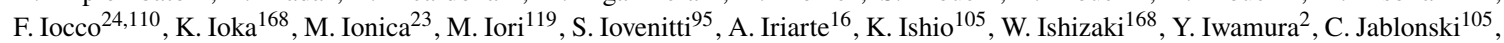
J. Jacquemier $^{45}$, M. Jacquemont ${ }^{45}$, M. Jamrozy ${ }^{169}$, P. Janecek ${ }^{33}$, F. Jankowsky ${ }^{170}$, A. Jardin-Blicq ${ }^{31}$, C. Jarnot ${ }^{87}$, P. Jean ${ }^{87}$, I. Jiménez Martínez $^{113}$, W. Jin ${ }^{171}$, L. Jocou ${ }^{125}$, N. Jordana ${ }^{172}$, M. Josselin ${ }^{73}$, L. Jouvin ${ }^{41}$, I. Jung-Richardt ${ }^{142}$, F.J.P.A. Junqueira ${ }^{19}$, C. JuramyGilles $^{79}$, J. Jurysek ${ }^{38}$, P. Kaaret ${ }^{173}$, L.H.S. Kadowaki ${ }^{19}$, M. Kagaya ${ }^{2}$, O. Kalekin ${ }^{142}$, R. Kankanyan ${ }^{53}$, D. Kantzas ${ }^{174}$, V. Karas ${ }^{34}$, A. Karastergiou ${ }^{114}$, S. $\operatorname{Karkar}^{79}$, E. Kasai ${ }^{48}$, J. Kasperek ${ }^{175}$, H. Katagiri ${ }^{176}$, J. Kataoka ${ }^{177}$, K. Katarzyński ${ }^{178}$, S. Katsuda ${ }^{179}$, U. Katz ${ }^{142}$, N. Kawanaka ${ }^{180}$, D. Kazanas ${ }^{130}$, D. Kerszberg ${ }^{41}$, B. Khélifi ${ }^{85}$, M.C. Kherlakian ${ }^{52}$, T.P. Kian ${ }^{181}$, D.B. Kieda ${ }^{164}$, T. Kihm ${ }^{53}$, S. Kim ${ }^{3}$, S. Kimeswenger ${ }^{163}$, S. Kisaka ${ }^{140}$, R. Kissmann ${ }^{163}$, R. Kleijwegt ${ }^{135}$, T. Kleiner ${ }^{52}$, G. Kluge ${ }^{10}$, W. Kluźniak ${ }^{49}$, J. Knapp ${ }^{52}$, J. Knödlseder ${ }^{87}$, A. Kobakhidze ${ }^{78}$, Y. Kobayashi ${ }^{2}$, B. Koch ${ }^{3}$, J. Kocot ${ }^{152}$, K. Kohri ${ }^{182}$, K. Kokkotas ${ }^{69}$, N. Komin ${ }^{58}$, A. Kong ${ }^{2}$, K. Kosack ${ }^{4}$, G. Kowal ${ }^{132}$, F. Krack ${ }^{52}$, M. Krause ${ }^{52}$, F. Krennrich ${ }^{129}$, M. Krumholz ${ }^{70}$, H. Kubo ${ }^{180}$, V. Kudryavtsev ${ }^{183}$, S. Kunwar ${ }^{53}$, Y. Kuroda ${ }^{139}$, J. Kushida ${ }^{157}$, P. Kushwaha ${ }^{19}$, A. La Barbera ${ }^{91}$, N. La Palombara ${ }^{61}$, V. La Parola ${ }^{91}$, G. La Rosa ${ }^{91}$, R. Lahmann ${ }^{142}$, G. Lamanna ${ }^{45}$, A. Lamastra ${ }^{28}$, M. Landoni ${ }^{95}$, D. Landriu ${ }^{4}$, R.G. Lang ${ }^{80}$, J. Lapington ${ }^{124}$, P. Laporte ${ }^{20}$, P. Lason ${ }^{152}$, J. Lasuik ${ }^{37}$, J. Lazendic-Galloway ${ }^{7}$, T. Le Flour ${ }^{45}$, P. Le Sidaner ${ }^{20}$, S. Leach ${ }^{124}$, A. Leckngam ${ }^{31}$, S.-H. Lee ${ }^{180}$, W.H. Lee ${ }^{16}$, S. Lee ${ }^{118}$, M.A. Leigui de Oliveira $^{184}$, A. Lemière ${ }^{85}$, M. Lemoine-Goumard ${ }^{106}$, J.-P. Lenain ${ }^{79}$, F. Leone ${ }^{94,185}$, V. Leray ${ }^{8}$, G. Leto ${ }^{29}$, F. Leuschner ${ }^{69}$, C. Levy ${ }^{79,20}$, R. Lindemann ${ }^{52}$, E. Lindfors ${ }^{65}$, L. Linhoff ${ }^{46}$, I. Liodakis ${ }^{65}$, A. Lipniacka ${ }^{116}$, S. Lloyd ${ }^{5}$, M. Lobo ${ }^{113}$, T. Lohse ${ }^{186}$, S. Lombardi ${ }^{28}$, F. Longo ${ }^{145}$, A. Lopez ${ }^{32}$, M. López ${ }^{11}$, R. López-Coto ${ }^{55}$, S. Loporchio ${ }^{149}$, F. Louis ${ }^{75}$, M. Louys ${ }^{20}$, F. Lucarelli ${ }^{28}$, D. Lucchesi ${ }^{55}$, H. Ludwig Boudi ${ }^{39}$, P.L. Luque-Escamilla ${ }^{56}$, E. Lyard ${ }^{38}$, M.C. Maccarone ${ }^{91}$, T. Maccarone ${ }^{187}$, E. Mach ${ }^{101}$, A.J. Maciejewski ${ }^{188}$, J. Mackey ${ }^{15}$, G.M. Madejski ${ }^{96}$, P. Maeght ${ }^{39}$, C. Maggio ${ }^{138}$, G. Maier ${ }^{52}$, A. Majczyna ${ }^{126}$, P. Majumdar ${ }^{83,2}$, M. Makariev ${ }^{189}$, M. Mallamaci ${ }^{55}$, R. Malta Nunes de Almeida ${ }^{184}$, S. Maltezos ${ }^{134}$, D. Malyshev ${ }^{142}$, D. Malyshev ${ }^{69}$, D. Mandat ${ }^{33}$, G. Maneva ${ }^{189}$, M. Manganaro ${ }^{121}$, G. Manicò ${ }^{94}$, P. Manigot ${ }^{8}$, K. Mannheim ${ }^{122}$, N. Maragos ${ }^{134}$, D. Marano ${ }^{29}$, M. Marconi ${ }^{84}$, A. Marcowith ${ }^{39}$, M. Marculewicz ${ }^{190}$, B. Marčun ${ }^{68}$, J. Marín ${ }^{98}$, N. Marinello ${ }^{55}$, P. Marinos ${ }^{118}$, M. Mariotti ${ }^{55}$, S. Markoff ${ }^{174}$, P. Marquez ${ }^{41}$, G. Marsella ${ }^{94}$, J. Martî ${ }^{56}$, J.-M. Martin ${ }^{20}$, P. Martin ${ }^{87}$, O. Martinez ${ }^{30}$, M. Martínez ${ }^{41}$, G. Martínez ${ }^{113}$, O. Martínez ${ }^{41}$, H. Martínez-Huerta ${ }^{80}$, C. Marty $^{87}$, R. Marx ${ }^{53}$, N. Masetti ${ }^{21,151}$, P. Massimino ${ }^{29}$, A. Mastichiadis ${ }^{191}$, H. Matsumoto ${ }^{167}$, N. Matthews ${ }^{164}$, G. Maurin ${ }^{45}$, W. Max-Moerbeck ${ }^{192}$, N. Maxted $^{43}$, D. Mazin²,105, M.N. Mazziotta ${ }^{120}$, S.M. Mazzola ${ }^{77}$, J.D. Mbarubucyeye ${ }^{52}$, L. Mc Comb ${ }^{5}$, I. McHardy ${ }^{115}$, S. McKeague ${ }^{107}$, S. McMuldroch ${ }^{63}$, E. Medina ${ }^{64}$, D. Medina Miranda ${ }^{17}$, A. Melandri ${ }^{95}$, C. Melioli $^{19}$, D. Melkumyan ${ }^{52}$, S. Menchiari ${ }^{62}$, S. Mender ${ }^{46}$, S. Mereghetti ${ }^{61}$, G. Merino Arévalo ${ }^{6}$, E. Mestre ${ }^{13}$, J.-L. Meunier ${ }^{79}$, T. Meures ${ }^{135}$, M. Meyer ${ }^{142}$, S. Micanovic ${ }^{121}$, M. Miceli ${ }^{77}$, M. Michailidis ${ }^{69}$, J. Michałowski ${ }^{101}$, T. Miener ${ }^{11}$, I. Mievre ${ }^{45}$, J. Miller ${ }^{35}$, I.A. Minaya ${ }^{153}$, T. Mineo ${ }^{91}$, M. Minev ${ }^{189}$, J.M. Miranda ${ }^{30}$, R. Mirzoyan ${ }^{105}$, A. Mitchel1 ${ }^{36}$, T. Mizuno ${ }^{193}$, B. Mode ${ }^{135}$, R. Moderski ${ }^{49}$, L. Mohrmann ${ }^{142}$, E. Molina ${ }^{81}$, E. Molinari ${ }^{148}$, T. Montaruli ${ }^{17}$, I. Monteiro ${ }^{45}$, C. Moore ${ }^{124}$, A. Moralejo ${ }^{41}$, D. Morcuende-Parrilla ${ }^{11}$, E. Moretti ${ }^{41}$, L. Morganti ${ }^{64}$, K. Mori ${ }^{194}$, P. Moriarty ${ }^{15}$, K. Morik ${ }^{46}$, G. Morlino ${ }^{22}$, P. Morris ${ }^{14}$, A. Morselli ${ }^{25}$, K. Mosshammer ${ }^{52}$, P. Moya ${ }^{192}$, R. Mukherjee ${ }^{9}$, J. Muller $^{8}$, C. Mundell ${ }^{172}$, J. Mundet ${ }^{41}$, T. Murach ${ }^{52}$, A. Muraczewski ${ }^{49}$, H. Muraishi ${ }^{195}$, K. Murase ${ }^{2}$, I. Musella ${ }^{84}$, A. Musumarra ${ }^{120}$, A. Nagai ${ }^{17}$, N. Nagar ${ }^{196}$, S. Nagataki ${ }^{54}$, T. Naito ${ }^{156}$, T. Nakamori ${ }^{154}$, K. Nakashima ${ }^{142}$, K. Nakayama ${ }^{51}$, N. Nakhjiri ${ }^{13}$, G. Naletto ${ }^{55}$ D. Naumann ${ }^{52}$, L. Nava ${ }^{95}$, R. Navarro ${ }^{174}$, M.A. Nawaz ${ }^{132}$, H. Ndiyavala ${ }^{1}$, D. Neise ${ }^{36}$, L. Nellen ${ }^{16}$, R. Nemmen ${ }^{19}$, M. Newbold ${ }^{164}$, N. Neyroud ${ }^{45}$, K. Ngernphat ${ }^{31}$, T. Nguyen Trung ${ }^{73}$, L. Nicastro ${ }^{21}$, L. Nickel ${ }^{46}$, J. Niemiec ${ }^{101}$, D. Nieto ${ }^{11}$, M. Nievas $^{32}$, C. Nigro $^{41}$, M. Nikołajuk ${ }^{190}$, D. Ninci ${ }^{41}$, K. Nishijima ${ }^{157}$, K. Noda ${ }^{2}$, Y. Nogami ${ }^{176}$, S. Nolan ${ }^{5}$, R. Nomura ${ }^{2}$, R. Norris ${ }^{117}$, D. Nosek ${ }^{197}$, M. Nöthe ${ }^{46}$, B. Novosyadlyj ${ }^{198}$, V. Novotny ${ }^{197}$, S. Nozaki ${ }^{180}$, F. Nunio ${ }^{144}$, P. O’Brien ${ }^{124}$, K. Obara ${ }^{176}$, R. Oger ${ }^{85}$, Y. Ohira ${ }^{51}$, M. Ohishi ${ }^{2}$, S. Ohm ${ }^{52}$, Y. Ohtani ${ }^{2}$, T. Oka ${ }^{180}$, N. Okazaki ${ }^{2}$, A. Okumura ${ }^{139,199}$, J.-F. Olive ${ }^{87}$, C. Oliver ${ }^{30}$, G. Olivera ${ }^{52}$, B. Olmi ${ }^{22}$, R.A. Ong ${ }^{71}$, M. Orienti ${ }^{90}$, R. Orito ${ }^{200}$, M. Orlandini ${ }^{21}$, S. Orlando ${ }^{77}$, E. Orlando ${ }^{145}$, J.P. Osborne ${ }^{124}$, M. Ostrowski ${ }^{169}$, N. Otte ${ }^{146}$, E. Ovcharov ${ }^{86}$, E. Owen ${ }^{2}$, I. Oya ${ }^{159}$, A. Ozieblo ${ }^{152}$, M. Padovani ${ }^{22}$, I. Pagano ${ }^{29}$, A. Pagliaro ${ }^{91}$, A. Paizis ${ }^{61}$, M. Palatiello ${ }^{145}$, M. Palatka ${ }^{33}$, E. Palazzi ${ }^{21}$, J.-L. Panazol ${ }^{45}$, D. Paneque ${ }^{105}$, B. Panes $^{3}$, S. Panny ${ }^{163}$, F.R. Pantaleo ${ }^{72}$, M. Panter ${ }^{53}$, R. Paoletti ${ }^{62}$, M. Paolillo ${ }^{24,110}$, A. Papitto ${ }^{28}$, A. Paravac ${ }^{122}$, J.M. Paredes ${ }^{81}$, G. Pareschi ${ }^{95}$, N. Park ${ }^{127}$, N. Parmiggiani ${ }^{21}$, R.D. Parsons ${ }^{186}$, P. Paśko ${ }^{201}$, S. Patel ${ }^{52}$, B. Patricellii ${ }^{28}$, G. Pauletta ${ }^{103}$, L. Pavletić ${ }^{121}$, S. Pavy ${ }^{8}$, A. Pe'er ${ }^{105}$, M. Pech ${ }^{33}$, M. Pecimotika ${ }^{121}$, M.G. Pellegriti ${ }^{120}$, P. Peñil Del $\mathrm{Campo}^{11}$, M. Penno ${ }^{52}$, A. Pepato ${ }^{55}$, S. Perard ${ }^{106}$, C. Perennes ${ }^{55}$, G. Peres ${ }^{77}$, M. Peresano ${ }^{4}$, A. Pérez-Aguilera ${ }^{11}$, J. Pérez-Romero ${ }^{14}$, M.A. Pérez-Torres ${ }^{12}$, M. Perri ${ }^{28}$, M. Persic ${ }^{103}$, S. Petrera ${ }^{18}$, P.-O. Petrucci ${ }^{125}$, O. Petruk ${ }^{66}$, B. Peyaud ${ }^{89}$, K. Pfrang ${ }^{52}$, E. Pian ${ }^{21}$, G. Piano ${ }^{99}$, P. Piatteli ${ }^{94}$, E. Pietropaolo ${ }^{18}$, R. Pillera ${ }^{149}$, B. Pilszyk ${ }^{101}$, D. Pimentel ${ }^{202}$, F. Pintore ${ }^{91}$, C. Pio García ${ }^{41}$, G. Pirola ${ }^{64}$, F. Piron ${ }^{39}$, A. Pisarski ${ }^{190}$, S. Pita ${ }^{85}$, M. Pohl ${ }^{128}$, V. Poireau ${ }^{45}$, P. Poledrelli ${ }^{159}$, A. Pollo ${ }^{126}$, M. Polo ${ }^{113}$, C. Pongkitivanichkul ${ }^{31}$,

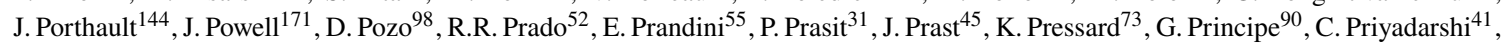
N. Produit ${ }^{38}$, D. Prokhorov ${ }^{174}$, H. Prokoph ${ }^{52}$, M. Prouza ${ }^{33}$, H. Przybilski $^{101}$, E. Pueschel ${ }^{52}$, G. Pühlhofer ${ }^{69}$, I. Puljak ${ }^{150}$, M.L. Pumo ${ }^{94}$, M. Punch ${ }^{85,57}$, F. Queiroz ${ }^{203}$, J. Quinn ${ }^{204}$, A. Quirrenbach ${ }^{170}$, S. Rainò ${ }^{149}$, P.J. Rajda ${ }^{175}$, R. Rando ${ }^{55}$, S. Razzaque ${ }^{205}$, E. Rebert ${ }^{20}$, S. Recchia ${ }^{85}$, P. Reichherzer ${ }^{59}$, O. Reimer ${ }^{163}$, A. Reimer ${ }^{163}$, A. Reisenegger ${ }^{3,206}$, Q. Remy ${ }^{53}$, M. Renaud ${ }^{39}$, T. Reposeur ${ }^{106}$, B. Reville ${ }^{53}$, J.-M. Reymond ${ }^{75}$, J. Reynolds ${ }^{15}$, W. Rhode ${ }^{46}$, D. Ribeiro ${ }^{9}$, M. Ribó ${ }^{81}$, G. Richards ${ }^{162}$, T. Richtler ${ }^{196}$, J. Rico ${ }^{41}$, F. Rieger ${ }^{53}$, L. Riitano ${ }^{135}$, V. Ripepi ${ }^{84}$, M. Riquelme ${ }^{192}$, D. Riquelme ${ }^{35}$, S. Rivoire ${ }^{39}$, V. Rizi ${ }^{18}$, E. Roache ${ }^{63}$, B. Röben ${ }^{159}$, M. Roche ${ }^{106}$, J. Rodriguez ${ }^{4}$, G. Rodriguez Fernandez ${ }^{25}$, J.C. Rodriguez Ramirez ${ }^{19}$, J.J. Rodríguez Vázquez ${ }^{113}$, F. Roepke ${ }^{170}$, G. $\operatorname{Rojas}^{207}$, L. Romanato ${ }^{55}$, P. Romano ${ }^{95}$, G. Romeo ${ }^{29}$, F. Romero Lobato ${ }^{11}$, C. Romoli ${ }^{53}$, M. Roncadelli ${ }^{103}$, S. Ronda ${ }^{30}$, J. Rosado ${ }^{11}$, 
A. Rosales de Leon $^{5}$, G. Rowell ${ }^{118}$, B. Rudak ${ }^{49}$, A. Rugliancich ${ }^{74}$, J.E. Ruíz del Mazo ${ }^{12}$, W. Rujopakarn ${ }^{31}$, C. Rulten ${ }^{5}$, C. Russell ${ }^{3}$, F. Russo ${ }^{21}$, I. Sadeh ${ }^{52}$, E. Sæther Hatlen ${ }^{10}$, S. Safi-Harb ${ }^{37}$, L. Saha ${ }^{11}$, P. Saha ${ }^{208}$, V. Sahakian ${ }^{147}$, S. Sailer ${ }^{53}$, T. Saito ${ }^{2}$, N. Sakaki ${ }^{54}$, S. Sakurai ${ }^{2}$, F. Salesa Greus ${ }^{101}$, G. Salina ${ }^{25}$, H. Salzmann ${ }^{69}$, D. Sanchez ${ }^{45}$, M. Sánchez-Conde ${ }^{14}$, H. Sandaker ${ }^{10}$, A. Sandoval ${ }^{16}$, P. Sangiorgi ${ }^{91}$, M. Sanguillon ${ }^{39}$, H. Sano ${ }^{2}$, M. Santander ${ }^{171}$, A. Santangelo ${ }^{69}$, E.M. Santos ${ }^{202}$, R. Santos-Lima ${ }^{19}$, A. Sanuy ${ }^{81}$, L. Sapozhnikov ${ }^{96}$, T. Saric ${ }^{150}$, S. Sarkar ${ }^{14}$, H. Sasaki ${ }^{157}$, N. Sasaki ${ }^{179}$, K. Satalecka ${ }^{52}$, Y. Sato ${ }^{209}$, F.G. Saturni ${ }^{28}$, M. Sawada ${ }^{54}$, U. Sawangwit ${ }^{31}$, J. Schaefer ${ }^{142}$, A. Scherer ${ }^{3}$, J. Scherpenberg ${ }^{105}$, P. Schipani ${ }^{84}$, B. Schleicher ${ }^{122}$, J. Schmoll ${ }^{5}$, M. Schneider ${ }^{143}$, H. Schoorlemmer ${ }^{53}$, P. Schovanek ${ }^{33}$, F. Schussler ${ }^{89}$, B. Schwab ${ }^{142}$, U. Schwanke ${ }^{186}$, J. Schwarz ${ }^{95}$, T. Schweizer ${ }^{105}$, E. Sciacca ${ }^{29}$, S. Scuderi ${ }^{61}$, M. Seglar Arroyo ${ }^{45}$, A. Segreto ${ }^{91}$, I. Seitenzahl ${ }^{43}$, D. Semikoz ${ }^{85}$, O. Sergijenko ${ }^{136}$, J.E. Serna Franco ${ }^{16}$, M. Servillat ${ }^{20}$, K. Seweryn ${ }^{201}$, V. Sguera ${ }^{21}$, A. Shalchi ${ }^{37}$, R.Y. Shang ${ }^{71}$, P. Sharma ${ }^{73}$, R.C. Shellard ${ }^{40}$, L. Sidoli ${ }^{61}$, J. Sieiro ${ }^{81}$, H. Siejkowski ${ }^{152}$, J. Silk ${ }^{114}$, A. Sillanpää 65 , B.B. Singh ${ }^{109}$, K.K. Singh ${ }^{210}$, A. Sinha ${ }^{39}$, C. Siqueira ${ }^{80}$, G. Sironi ${ }^{95}$, J. Sitarek ${ }^{60}$, P. Sizun ${ }^{75}$, V. Sliusar ${ }^{38}$, A. Slowikowska ${ }^{178}$, D. Sobczyńska ${ }^{60}$, R.W. Sobrinho ${ }^{184}$, H. Sol $^{20}$, G. Sottile ${ }^{91}$, H. Spackman ${ }^{114}$, A. Specovius ${ }^{142}$, S. Spencer ${ }^{114}$, G. Spengler ${ }^{186}$, D. Spiga ${ }^{95}$, A. Spolon ${ }^{55}$, W. Springer ${ }^{164}$, A. Stamerra ${ }^{28}$, S. Stanič ${ }^{68}$, R. Starling ${ }^{124}$, Ł. Stawarz ${ }^{169}$, R. Steenkamp ${ }^{48}$, S. Stefanik ${ }^{197}$, C. Stegmann ${ }^{128}$, A. Steiner ${ }^{52}$, S. Steinmass ${ }^{53}$, C. Stella ${ }^{103}$, C. Steppa ${ }^{128}$, R. Sternberger ${ }^{52}$, M. Sterzel ${ }^{152}$, C. Stevens ${ }^{135}$, B. Stevenson ${ }^{71}$, T. Stolarczyk ${ }^{4}$, G. Stratta ${ }^{21}$, U. Straumann ${ }^{208}$, J. Strišković ${ }^{166}$, M. Strzys ${ }^{2}$, R. Stuik ${ }^{174}$, M. Suchenek ${ }^{211}$, Y. Suda ${ }^{140}$, Y. Sunada $^{179}$, T. Suomijarvi ${ }^{73}$, T. Suric ${ }^{212}$, P. Sutcliffe ${ }^{153}$, H. Suzuki $^{213}$, P. Świerk ${ }^{101}$, T. Szepieniec ${ }^{152}$, A. Tacchini ${ }^{21}$, K. Tachihara ${ }^{141}$, G. Tagliaferri ${ }^{95}$, H. Tajima ${ }^{139}$, N. Tajima ${ }^{2}$, D. Tak ${ }^{52}$, K. Takahashi ${ }^{214}$, H. Takahashi ${ }^{140}$, M. Takahashi ${ }^{2}$, M. Takahashi ${ }^{2}$, J. Takata $^{2}$,

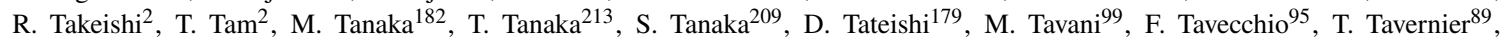
L. Taylor ${ }^{135}$, A. Taylor ${ }^{52}$, L.A. Tejedor ${ }^{11}$, P. Temnikov ${ }^{189}$, Y. Terada ${ }^{179}$, K. Terauchi ${ }^{180}$, J.C. Terrazas ${ }^{192}$, R. Terrier $^{85}$, T. Terzic ${ }^{121}$, M. Teshima ${ }^{105,2}$, V. Testa ${ }^{28}$, D. Thibaut ${ }^{85}$, F. Thocquenne ${ }^{75}$, W. Tian $^{2}$, L. Tibaldo ${ }^{87}$, A. Tiengo ${ }^{215}$, D. Tiziani ${ }^{142}$, M. Tluczykont ${ }^{50}$, C.J. Todero Peixoto ${ }^{102}$, F. Tokanai ${ }^{154}$, K. Toma ${ }^{160}$, L. Tomankova ${ }^{142}$, J. Tomastik ${ }^{104}$, D. Tonev ${ }^{189}$, M. Tornikoski ${ }^{216}$, D.F. Torres ${ }^{13}$, E. Torresi ${ }^{21}$, G. Tosti ${ }^{95}$, L. Tosti ${ }^{23}$, T. Totani ${ }^{51}$, N. Tothill ${ }^{117}$, F. Toussenel ${ }^{79}$, G. Tovmassian ${ }^{16}$, P. Travnicek ${ }^{33}$, C. Trichard ${ }^{8}$,

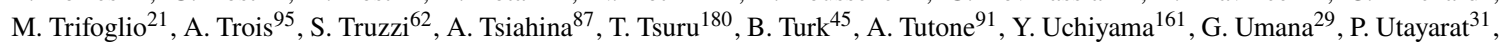
L. Vaclavek ${ }^{104}$, M. Vacula ${ }^{104}$, V. Vagelli ${ }^{23,217}$, F. Vagnetti ${ }^{25}$, F. Vakili ${ }^{218}$, J.A. Valdivia ${ }^{192}$, M. Valentino ${ }^{24}$, A. Valio ${ }^{19}$, B. Vallage ${ }^{89}$, P. Vallania ${ }^{44,64}$, J.V. Valverde Quispe ${ }^{8}$, A.M. Van den Berg ${ }^{42}$, W. van Driel ${ }^{20}$, C. van Eldik ${ }^{142}$, C. van Rensburg ${ }^{1}$, B. van Soelen ${ }^{210}$, J. Vandenbroucke ${ }^{135}$, J. Vanderwalt ${ }^{1}$, G. Vasileiadis ${ }^{39}$, V. Vassiliev ${ }^{71}$, M. Vázquez Acosta ${ }^{32}$, M. Vecchi ${ }^{42}$, A. Vega ${ }^{9}$, J. Veh $^{142}$, P. Veitch ${ }^{118}$, P. Venault ${ }^{75}$, C. Venter ${ }^{1}$, S. Ventura ${ }^{62}$, S. Vercellone ${ }^{95}$, S. Vergani ${ }^{20}$, V. Verguilov ${ }^{189}$, G. Verna $^{27}$, S. Vernetto ${ }^{44,64}$, V. Verzi ${ }^{25}$, G.P. Vettolani ${ }^{90}$, C. Veyssiere ${ }^{144}$, I. Viale ${ }^{55}$, A. Viana ${ }^{80}$, N. Viaux ${ }^{35}$, J. Vicha ${ }^{33}$, J. Vignatti ${ }^{35}$, C.F. Vigorito ${ }^{64,108}$, J. Villanueva ${ }^{98}$, J. Vink ${ }^{174}$, V. Vitale ${ }^{23}$, V. Vittorini ${ }^{99}$, V. Vodeb ${ }^{68}$, H. Voelk ${ }^{53}$, N. Vogel ${ }^{142}$, V. Voisin ${ }^{79}$, S. Vorobiov ${ }^{68}$, I. Vovk ${ }^{2}$, M. Vrastil ${ }^{33}$, T. Vuillaume ${ }^{45}$, S.J. Wagner ${ }^{170}$, R. Wagner ${ }^{105}$, P. Wagner ${ }^{52}$, K. Wakazono ${ }^{139}$, S.P. Wakely ${ }^{127}$, R. Walter ${ }^{38}$, M. Ward ${ }^{5}$, D. Warren ${ }^{54}$, J. Watson ${ }^{52}$, N. Webb ${ }^{87}$, M. Wechakama ${ }^{31}$, P. Wegner ${ }^{52}$, A. Weinstein ${ }^{129}$, C. Weniger ${ }^{174}$, F. Werner ${ }^{53}$, H. Wetteskind ${ }^{105}$, M. White ${ }^{118}$, R. White ${ }^{53}$, A. Wierzcholska ${ }^{101}$, S. Wiesand ${ }^{52}$, R. Wijers ${ }^{174}$, M. Wilkinson ${ }^{124}$, M. Will ${ }^{105}$, D.A. Williams ${ }^{143}$, J. Williams ${ }^{124}$, T. Williamson ${ }^{162}$, A. Wolter ${ }^{95}$, Y.W. Wong ${ }^{142}$, M. Wood ${ }^{96}$, C. Wunderlich ${ }^{62}$, T. Yamamoto ${ }^{213}$, H. Yamamoto ${ }^{141}$,

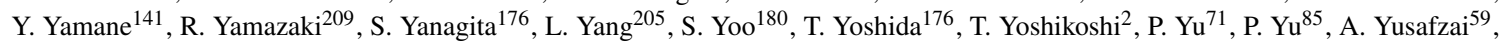
M. Zacharias ${ }^{20}$, G. Zaharijas ${ }^{68}$, B. Zaldivar ${ }^{14}$, L. Zampieri ${ }^{76}$, R. Zanmar Sanchez ${ }^{29}$, D. Zaric ${ }^{150}$, M. Zavrtanik ${ }^{68}$, D. Zavrtanik ${ }^{68}$, A.A. Zdziarski ${ }^{49}$, A. Zech ${ }^{20}$, H. Zechlin ${ }^{64}$, A. Zenin ${ }^{139}$, A. Zerwekh ${ }^{35}$, V.I. Zhdanov ${ }^{136}$, K. Ziętara ${ }^{169}$, A. Zink ${ }^{142}$, J. Ziółkowski ${ }^{49}$, V. Zitelli ${ }^{21}$, M. Živec ${ }^{68}$, A. Zmija ${ }^{142}$

1: Centre for Space Research, North-West University, Potchefstroom, 2520, South Africa

2: Institute for Cosmic Ray Research, University of Tokyo, 5-1-5, Kashiwa-no-ha, Kashiwa, Chiba 277-8582, Japan

3: Pontificia Universidad Católica de Chile, Av. Libertador Bernardo O’Higgins 340, Santiago, Chile

4 : AIM, CEA, CNRS, Université Paris-Saclay, Université Paris Diderot, Sorbonne Paris Cité, CEA Paris-Saclay, IRFU/DAp,

Bat 709, Orme des Merisiers, 91191 Gif-sur-Yvette, France

5: Centre for Advanced Instrumentation, Dept. of Physics, Durham University, South Road, Durham DH1 3LE, United Kingdom

6: Port d'Informació Científica, Edifici D, Carrer de l'Albareda, 08193 Bellaterrra (Cerdanyola del Vallès), Spain

7: School of Physics and Astronomy, Monash University, Melbourne, Victoria 3800, Australia

8 : Laboratoire Leprince-Ringuet, École Polytechnique (UMR 7638, CNRS/IN2P3, Institut Polytechnique de Paris), 91128 Palaiseau, France

9: Department of Physics, Columbia University, 538 West 120th Street, New York, NY 10027, USA

10 : University of Oslo, Department of Physics, Sem Saelandsvei 24 - PO Box 1048 Blindern, N-0316 Oslo, Norway

11 : EMFTEL department and IPARCOS, Universidad Complutense de Madrid, 28040 Madrid, Spain

12 : Instituto de Astrofísica de Andalucía-CSIC, Glorieta de la Astronomía s/n, 18008, Granada, Spain

13 : Institute of Space Sciences (ICE-CSIC), and Institut d'Estudis Espacials de Catalunya (IEEC), and Institució Catalana de Recerca I Estudis Avançats (ICREA), Campus UAB, Carrer de Can Magrans, s/n 08193 Cerdanyola del Vallés, Spain

14 : Instituto de Física Teórica UAM/CSIC and Departamento de Física Teórica, Universidad Autónoma de Madrid, c/ Nicolás

Cabrera 13-15, Campus de Cantoblanco UAM, 28049 Madrid, Spain

15 : Dublin Institute for Advanced Studies, 31 Fitzwilliam Place, Dublin 2, Ireland

16: Universidad Nacional Autónoma de México, Delegación Coyoacán, 04510 Ciudad de México, Mexico

17 : University of Geneva - Département de physique nucléaire et corpusculaire, 24 rue du Général-Dufour, 1211 Genève 4, Switzerland

18: INFN Dipartimento di Scienze Fisiche e Chimiche - Università degli Studi dell'Aquila and Gran Sasso Science Institute, Via Vetoio 1, Viale Crispi 7, 67100 L’Aquila, Italy 
19: Instituto de Astronomia, Geofísico, e Ciências Atmosféricas - Universidade de São Paulo, Cidade Universitária, R. do Matão, 1226, CEP 05508-090, São Paulo, SP, Brazil

20 : LUTH, GEPI and LERMA, Observatoire de Paris, CNRS, PSL University, 5 place Jules Janssen, 92190, Meudon, France

21 : INAF - Osservatorio di Astrofisica e Scienza dello spazio di Bologna, Via Piero Gobetti 93/3, 40129 Bologna, Italy

22 : INAF - Osservatorio Astrofisico di Arcetri, Largo E. Fermi, 5 - 50125 Firenze, Italy

23 : INFN Sezione di Perugia and Università degli Studi di Perugia, Via A. Pascoli, 06123 Perugia, Italy

24 : INFN Sezione di Napoli, Via Cintia, ed. G, 80126 Napoli, Italy

25 : INFN Sezione di Roma Tor Vergata, Via della Ricerca Scientifica 1, 00133 Rome, Italy

26: Argonne National Laboratory, 9700 S. Cass Avenue, Argonne, IL 60439, USA

27: Aix-Marseille Université, CNRS/IN2P3, CPPM, 163 Avenue de Luminy, 13288 Marseille cedex 09, France

28: INAF - Osservatorio Astronomico di Roma, Via di Frascati 33, 00040, Monteporzio Catone, Italy

29: INAF - Osservatorio Astrofisico di Catania, Via S. Sofia, 78, 95123 Catania, Italy

30 : Grupo de Electronica, Universidad Complutense de Madrid, Av. Complutense s/n, 28040 Madrid, Spain

31 : National Astronomical Research Institute of Thailand, 191 Huay Kaew Rd., Suthep, Muang, Chiang Mai, 50200, Thailand

32: Instituto de Astrofísica de Canarias and Departamento de Astrofísica, Universidad de La Laguna, La Laguna, Tenerife, Spain

33 : FZU - Institute of Physics of the Czech Academy of Sciences, Na Slovance 1999/2, 18221 Praha 8, Czech Republic

34 : Astronomical Institute of the Czech Academy of Sciences, Bocni II 1401 - 14100 Prague, Czech Republic

35 : CCTVal, Universidad Técnica Federico Santa María, Avenida España 1680, Valparaíso, Chile

36 : ETH Zurich, Institute for Particle Physics, Schafmattstr. 20, CH-8093 Zurich, Switzerland

37 : The University of Manitoba, Dept of Physics and Astronomy, Winnipeg, Manitoba R3T 2N2, Canada

38 : Department of Astronomy, University of Geneva, Chemin d'Ecogia 16, CH-1290 Versoix, Switzerland

39: Laboratoire Univers et Particules de Montpellier, Université de Montpellier, CNRS/IN2P3, CC 72, Place Eugène Bataillon,

F-34095 Montpellier Cedex 5, France

40 : Centro Brasileiro de Pesquisas Físicas, Rua Xavier Sigaud 150, RJ 22290-180, Rio de Janeiro, Brazil

41 : Institut de Fisica d'Altes Energies (IFAE), The Barcelona Institute of Science and Technology, Campus UAB, 08193 Bellaterra (Barcelona), Spain

42: University of Groningen, KVI - Center for Advanced Radiation Technology, Zernikelaan 25, 9747 AA Groningen, The Netherlands

43 : School of Physics, University of New South Wales, Sydney NSW 2052, Australia

44 : INAF - Osservatorio Astrofisico di Torino, Strada Osservatorio 20, 10025 Pino Torinese (TO), Italy

45 : Univ. Savoie Mont Blanc, CNRS, Laboratoire d'Annecy de Physique des Particules - IN2P3, 74000 Annecy, France

46 : Department of Physics, TU Dortmund University, Otto-Hahn-Str. 4, 44221 Dortmund, Germany

47 : University of Zagreb, Faculty of electrical engineering and computing, Unska 3, 10000 Zagreb, Croatia

48 : University of Namibia, Department of Physics, 340 Mandume Ndemufayo Ave., Pioneerspark, Windhoek, Namibia

49 : Nicolaus Copernicus Astronomical Center, Polish Academy of Sciences, ul. Bartycka 18, 00-716 Warsaw, Poland

50 : Universität Hamburg, Institut für Experimentalphysik, Luruper Chaussee 149, 22761 Hamburg, Germany

51: Graduate School of Science, University of Tokyo, 7-3-1 Hongo, Bunkyo-ku, Tokyo 113-0033, Japan

52 : Deutsches Elektronen-Synchrotron, Platanenallee 6, 15738 Zeuthen, Germany

53: Max-Planck-Institut für Kernphysik, Saupfercheckweg 1, 69117 Heidelberg, Germany

54 : RIKEN, Institute of Physical and Chemical Research, 2-1 Hirosawa, Wako, Saitama, 351-0198, Japan

55 : INFN Sezione di Padova and Università degli Studi di Padova, Via Marzolo 8, 35131 Padova, Italy

56 : Escuela Politécnica Superior de Jaén, Universidad de Jaén, Campus Las Lagunillas s/n, Edif. A3, 23071 Jaén, Spain

57 : Department of Physics and Electrical Engineering, Linnaeus University, 35195 Växjö, Sweden

58: University of the Witwatersrand, 1 Jan Smuts Avenue, Braamfontein, 2000 Johannesburg, South Africa

59 : Institut für Theoretische Physik, Lehrstuhl IV: Plasma-Astroteilchenphysik, Ruhr-Universität Bochum, Universitätsstraße 150, 44801 Bochum, Germany

60 : Faculty of Physics and Applied Computer Science, University of Lódź, ul. Pomorska 149-153, 90-236 Lódź, Poland

61: INAF - Istituto di Astrofisica Spaziale e Fisica Cosmica di Milano, Via A. Corti 12, 20133 Milano, Italy

62 : INFN and Università degli Studi di Siena, Dipartimento di Scienze Fisiche, della Terra e dell'Ambiente (DSFTA), Sezione

di Fisica, Via Roma 56, 53100 Siena, Italy

63 : Center for Astrophysics | Harvard \& Smithsonian, 60 Garden St, Cambridge, MA 02180, USA

64 : INFN Sezione di Torino, Via P. Giuria 1, 10125 Torino, Italy

65 : Finnish Centre for Astronomy with ESO, University of Turku, Finland, FI-20014 University of Turku, Finland

66 : Pidstryhach Institute for Applied Problems in Mechanics and Mathematics NASU, 3B Naukova Street, Lviv, 79060, Ukraine

67 : Bhabha Atomic Research Centre, Trombay, Mumbai 400085, India

68 : Center for Astrophysics and Cosmology, University of Nova Gorica, Vipavska 11c, 5270 Ajdovščina, Slovenia

69: Institut für Astronomie und Astrophysik, Universität Tübingen, Sand 1, 72076 Tübingen, Germany

70 : Research School of Astronomy and Astrophysics, Australian National University, Canberra ACT 0200, Australia

71: Department of Physics and Astronomy, University of California, Los Angeles, CA 90095, USA

72: INFN Sezione di Bari and Politecnico di Bari, via Orabona 4, 70124 Bari, Italy 
73 : Laboratoire de Physique des 2 infinis, Irene Joliot-Curie,IN2P3/CNRS, Université Paris-Saclay, Université de Paris, 15 rue Georges Clemenceau, 91406 Orsay, Cedex, France

74 : INFN Sezione di Pisa, Largo Pontecorvo 3, 56217 Pisa, Italy

75 : IRFU/DEDIP, CEA, Université Paris-Saclay, Bat 141, 91191 Gif-sur-Yvette, France

76 : INAF - Osservatorio Astronomico di Padova, Vicolo dell’Osservatorio 5, 35122 Padova, Italy

77 : INAF - Osservatorio Astronomico di Palermo "G.S. Vaiana", Piazza del Parlamento 1, 90134 Palermo, Italy

78 : School of Physics, University of Sydney, Sydney NSW 2006, Australia

79: Sorbonne Université, Université Paris Diderot, Sorbonne Paris Cité, CNRS/IN2P3, Laboratoire de Physique Nucléaire et de Hautes Energies, LPNHE, 4 Place Jussieu, F-75005 Paris, France

80 : Instituto de Física de São Carlos, Universidade de São Paulo, Av. Trabalhador São-carlense, 400 - CEP 13566-590, São Carlos, SP, Brazil

81 : Departament de Física Quàntica i Astrofísica, Institut de Ciències del Cosmos, Universitat de Barcelona, IEEC-UB, Martí i Franquès, 1, 08028, Barcelona, Spain

82 : Department of Physics, Washington University, St. Louis, MO 63130, USA

83 : Saha Institute of Nuclear Physics, Bidhannagar, Kolkata-700 064, India

84 : INAF - Osservatorio Astronomico di Capodimonte, Via Salita Moiariello 16, 80131 Napoli, Italy

85: Université de Paris, CNRS, Astroparticule et Cosmologie, 10, rue Alice Domon et Léonie Duquet, 75013 Paris Cedex 13, France

86 : Astronomy Department of Faculty of Physics, Sofia University, 5 James Bourchier Str., 1164 Sofia, Bulgaria

87 : Institut de Recherche en Astrophysique et Planétologie, CNRS-INSU, Université Paul Sabatier, 9 avenue Colonel Roche, BP 44346, 31028 Toulouse Cedex 4, France

88 : School of Physics and Astronomy, University of Minnesota, 116 Church Street S.E. Minneapolis, Minnesota 55455-0112, USA

89: IRFU, CEA, Université Paris-Saclay, Bât 141, 91191 Gif-sur-Yvette, France

90 : INAF - Istituto di Radioastronomia, Via Gobetti 101, 40129 Bologna, Italy

91 : INAF - Istituto di Astrofisica Spaziale e Fisica Cosmica di Palermo, Via U. La Malfa 153, 90146 Palermo, Italy

92: Astronomical Observatory, Department of Physics, University of Warsaw, Aleje Ujazdowskie 4, 00478 Warsaw, Poland

93: Armagh Observatory and Planetarium, College Hill, Armagh BT61 9DG, United Kingdom

94 : INFN Sezione di Catania, Via S. Sofia 64, 95123 Catania, Italy

95 : INAF - Osservatorio Astronomico di Brera, Via Brera 28, 20121 Milano, Italy

96: Kavli Institute for Particle Astrophysics and Cosmology, Department of Physics and SLAC National Accelerator Laboratory, Stanford University, 2575 Sand Hill Road, Menlo Park, CA 94025, USA

97 : Universidade Cruzeiro do Sul, Núcleo de Astrofísica Teórica (NAT/UCS), Rua Galvão Bueno 8687, Bloco B, sala 16, Libertade 01506-000 - São Paulo, Brazil

98: Universidad de Valparaíso, Blanco 951, Valparaiso, Chile

99: INAF - Istituto di Astrofisica e Planetologia Spaziali (IAPS), Via del Fosso del Cavaliere 100, 00133 Roma, Italy

100 : Lund Observatory, Lund University, Box 43, SE-22100 Lund, Sweden

101 : The Henryk Niewodniczański Institute of Nuclear Physics, Polish Academy of Sciences, ul. Radzikowskiego 152, 31-342 Cracow, Poland

102 : Escola de Engenharia de Lorena, Universidade de São Paulo, Área I - Estrada Municipal do Campinho, s/nº, CEP 12602-810,

Pte. Nova, Lorena, Brazil

103: INFN Sezione di Trieste and Università degli Studi di Udine, Via delle Scienze 208, 33100 Udine, Italy

104 : Palacky University Olomouc, Faculty of Science, RCPTM, 17. listopadu 1192/12, 77146 Olomouc, Czech Republic

105 : Max-Planck-Institut für Physik, Föhringer Ring 6, 80805 München, Germany

106 : CENBG, Univ. Bordeaux, CNRS-IN2P3, UMR 5797, 19 Chemin du Solarium, CS 10120, F-33175 Gradignan Cedex, France

107 : Dublin City University, Glasnevin, Dublin 9, Ireland

108 : Dipartimento di Fisica - Universitá degli Studi di Torino, Via Pietro Giuria 1 - 10125 Torino, Italy

109: Tata Institute of Fundamental Research, Homi Bhabha Road, Colaba, Mumbai 400005, India

110 : Universitá degli Studi di Napoli "Federico II" - Dipartimento di Fisica "E. Pancini", Complesso universitario di Monte Sant'Angelo, Via Cintia - 80126 Napoli, Italy

111 : Oskar Klein Centre, Department of Physics, University of Stockholm, Albanova, SE-10691, Sweden

112 : Yale University, Department of Physics and Astronomy, 260 Whitney Avenue, New Haven, CT 06520-8101, USA

113 : CIEMAT, Avda. Complutense 40, 28040 Madrid, Spain

114 : University of Oxford, Department of Physics, Denys Wilkinson Building, Keble Road, Oxford OX1 3RH, United Kingdom

115 : School of Physics \& Astronomy, University of Southampton, University Road, Southampton SO17 1BJ, United Kingdom

116 : Department of Physics and Technology, University of Bergen, Museplass 1, 5007 Bergen, Norway

117 : Western Sydney University, Locked Bag 1797, Penrith, NSW 2751, Australia

118: School of Physical Sciences, University of Adelaide, Adelaide SA 5005, Australia

119 : INFN Sezione di Roma La Sapienza, P.le Aldo Moro, 2 - 00185 Roma, Italy

120 : INFN Sezione di Bari, via Orabona 4, 70126 Bari, Italy 
121 : University of Rijeka, Department of Physics, Radmile Matejcic 2, 51000 Rijeka, Croatia

122 : Institute for Theoretical Physics and Astrophysics, Universität Würzburg, Campus Hubland Nord, Emil-Fischer-Str. 31, 97074 Würzburg, Germany

123 : Universidade Federal Do Paraná - Setor Palotina, Departamento de Engenharias e Exatas, Rua Pioneiro, 2153, Jardim Dallas, CEP: 85950-000 Palotina, Paraná, Brazil

124 : Dept. of Physics and Astronomy, University of Leicester, Leicester, LE1 7RH, United Kingdom

125 : Univ. Grenoble Alpes, CNRS, IPAG, 414 rue de la Piscine, Domaine Universitaire, 38041 Grenoble Cedex 9, France

126 : National Centre for nuclear research (Narodowe Centrum Badań Jądrowych), Ul. Andrzeja Sołtana7, 05-400 Otwock, Świerk, Poland

127 : Enrico Fermi Institute, University of Chicago, 5640 South Ellis Avenue, Chicago, IL 60637, USA

128 : Institut für Physik \& Astronomie, Universität Potsdam, Karl-Liebknecht-Strasse 24/25, 14476 Potsdam, Germany

129 : Department of Physics and Astronomy, Iowa State University, Zaffarano Hall, Ames, IA 50011-3160, USA

130 : School of Physics, Aristotle University, Thessaloniki, 54124 Thessaloniki, Greece

131 : King's College London, Strand, London, WC2R 2LS, United Kingdom

132 : Escola de Artes, Ciências e Humanidades, Universidade de São Paulo, Rua Arlindo Bettio, CEP 03828-000, 1000 São Paulo, Brazil

133 : Dept. of Astronomy \& Astrophysics, Pennsylvania State University, University Park, PA 16802, USA

134 : National Technical University of Athens, Department of Physics, Zografos 9, 15780 Athens, Greece

135 : University of Wisconsin, Madison, 500 Lincoln Drive, Madison, WI, 53706, USA

136 : Astronomical Observatory of Taras Shevchenko National University of Kyiv, 3 Observatorna Street, Kyiv, 04053, Ukraine

137 : Department of Physics, Purdue University, West Lafayette, IN 47907, USA

138 : Unitat de Física de les Radiacions, Departament de Física, and CERES-IEEC, Universitat Autònoma de Barcelona, Edifici

C3, Campus UAB, 08193 Bellaterra, Spain

139 : Institute for Space-Earth Environmental Research, Nagoya University, Chikusa-ku, Nagoya 464-8601, Japan

140 : Department of Physical Science, Hiroshima University, Higashi-Hiroshima, Hiroshima 739-8526, Japan

141 : Department of Physics, Nagoya University, Chikusa-ku, Nagoya, 464-8602, Japan

142 : Friedrich-Alexander-Universität Erlangen-Nürnberg, Erlangen Centre for Astroparticle Physics (ECAP), Erwin-Rommel-Str.

1, 91058 Erlangen, Germany

143 : Santa Cruz Institute for Particle Physics and Department of Physics, University of California, Santa Cruz, 1156 High Street,

Santa Cruz, CA 95064, USA

144 : IRFU / DIS, CEA, Université de Paris-Saclay, Bat 123, 91191 Gif-sur-Yvette, France

145 : INFN Sezione di Trieste and Università degli Studi di Trieste, Via Valerio 2 I, 34127 Trieste, Italy

146: School of Physics \& Center for Relativistic Astrophysics, Georgia Institute of Technology, 837 State Street, Atlanta, Georgia, 30332-0430, USA

147 : Alikhanyan National Science Laboratory, Yerevan Physics Institute, 2 Alikhanyan Brothers St., 0036, Yerevan, Armenia

148 : INAF - Telescopio Nazionale Galileo, Roche de los Muchachos Astronomical Observatory, 38787 Garafia, TF, Italy

149 : INFN Sezione di Bari and Università degli Studi di Bari, via Orabona 4, 70124 Bari, Italy

150 : University of Split - FESB, R. Boskovica 32, 21000 Split, Croatia

151: Universidad Andres Bello, República 252, Santiago, Chile

152: Academic Computer Centre CYFRONET AGH, ul. Nawojki 11, 30-950 Cracow, Poland

153 : University of Liverpool, Oliver Lodge Laboratory, Liverpool L69 7ZE, United Kingdom

154 : Department of Physics, Yamagata University, Yamagata, Yamagata 990-8560, Japan

155 : Astronomy Department, Adler Planetarium and Astronomy Museum, Chicago, IL 60605, USA

156 : Faculty of Management Information, Yamanashi-Gakuin University, Kofu, Yamanashi 400-8575, Japan

157 : Department of Physics, Tokai University, 4-1-1, Kita-Kaname, Hiratsuka, Kanagawa 259-1292, Japan

158 : Centre for Astrophysics Research, Science \& Technology Research Institute, University of Hertfordshire, College Lane, Hertfordshire AL10 9AB, United Kingdom

159: Cherenkov Telescope Array Observatory, Saupfercheckweg 1, 69117 Heidelberg, Germany

160 : Tohoku University, Astronomical Institute, Aobaku, Sendai 980-8578, Japan

161 : Department of Physics, Rikkyo University, 3-34-1 Nishi-Ikebukuro, Toshima-ku, Tokyo, Japan

162 : Department of Physics and Astronomy and the Bartol Research Institute, University of Delaware, Newark, DE 19716, USA

163 : Institut für Astro- und Teilchenphysik, Leopold-Franzens-Universität, Technikerstr. 25/8, 6020 Innsbruck, Austria

164 : Department of Physics and Astronomy, University of Utah, Salt Lake City, UT 84112-0830, USA

165 : IMAPP, Radboud University Nijmegen, P.O. Box 9010, 6500 GL Nijmegen, The Netherlands

166 : Josip Juraj Strossmayer University of Osijek, Trg Ljudevita Gaja 6, 31000 Osijek, Croatia

167 : Department of Earth and Space Science, Graduate School of Science, Osaka University, Toyonaka 560-0043, Japan

168 : Yukawa Institute for Theoretical Physics, Kyoto University, Kyoto 606-8502, Japan

169 : Astronomical Observatory, Jagiellonian University, ul. Orla 171, 30-244 Cracow, Poland

170 : Landessternwarte, Zentrum für Astronomie der Universität Heidelberg, Königstuhl 12, 69117 Heidelberg, Germany

171 : University of Alabama, Tuscaloosa, Department of Physics and Astronomy, Gallalee Hall, Box 870324 Tuscaloosa, AL 35487-0324, USA 
172 : Department of Physics, University of Bath, Claverton Down, Bath BA2 7AY, United Kingdom

173 : University of Iowa, Department of Physics and Astronomy, Van Allen Hall, Iowa City, IA 52242, USA

174 : Anton Pannekoek Institute/GRAPPA, University of Amsterdam, Science Park 9041098 XH Amsterdam, The Netherlands

175 : Faculty of Computer Science, Electronics and Telecommunications, AGH University of Science and Technology, Kraków,

al. Mickiewicza 30, 30-059 Cracow, Poland

176 : Faculty of Science, Ibaraki University, Mito, Ibaraki, 310-8512, Japan

177 : Faculty of Science and Engineering, Waseda University, Shinjuku, Tokyo 169-8555, Japan

178 : Institute of Astronomy, Faculty of Physics, Astronomy and Informatics, Nicolaus Copernicus University in Toruń, ul. Grudziądzka 5, 87-100 Toruń, Poland

179 : Graduate School of Science and Engineering, Saitama University, 255 Simo-Ohkubo, Sakura-ku, Saitama city, Saitama 338-8570, Japan

180 : Division of Physics and Astronomy, Graduate School of Science, Kyoto University, Sakyo-ku, Kyoto, 606-8502, Japan

181 : Centre for Quantum Technologies, National University Singapore, Block S15, 3 Science Drive 2, Singapore 117543 , Singapore

182 : Institute of Particle and Nuclear Studies, KEK (High Energy Accelerator Research Organization), 1-1 Oho, Tsukuba, 305-0801, Japan

183 : Department of Physics and Astronomy, University of Sheffield, Hounsfield Road, Sheffield S3 7RH, United Kingdom

184 : Centro de Ciências Naturais e Humanas, Universidade Federal do ABC, Av. dos Estados, 5001, CEP: 09.210-580, Santo André - SP, Brazil

185 : Dipartimento di Fisica e Astronomia, Sezione Astrofisica, Universitá di Catania, Via S. Sofia 78, I-95123 Catania, Italy

186 : Department of Physics, Humboldt University Berlin, Newtonstr. 15, 12489 Berlin, Germany

187: Texas Tech University, 2500 Broadway, Lubbock, Texas 79409-1035, USA

188 : University of Zielona Góra, ul. Licealna 9, 65-417 Zielona Góra, Poland

189 : Institute for Nuclear Research and Nuclear Energy, Bulgarian Academy of Sciences, 72 boul. Tsarigradsko chaussee, 1784

Sofia, Bulgaria

190 : University of Białystok, Faculty of Physics, ul. K. Ciołkowskiego 1L, 15-254 Białystok, Poland

191 : Faculty of Physics, National and Kapodestrian University of Athens, Panepistimiopolis, 15771 Ilissia, Athens, Greece

192 : Universidad de Chile, Av. Libertador Bernardo O’Higgins 1058, Santiago, Chile

193 : Hiroshima Astrophysical Science Center, Hiroshima University, Higashi-Hiroshima, Hiroshima 739-8526, Japan

194 : Department of Applied Physics, University of Miyazaki, 1-1 Gakuen Kibana-dai Nishi, Miyazaki, 889-2192, Japan

195 : School of Allied Health Sciences, Kitasato University, Sagamihara, Kanagawa 228-8555, Japan

196 : Departamento de Astronomía, Universidad de Concepción, Barrio Universitario S/N, Concepción, Chile

197 : Charles University, Institute of Particle \& Nuclear Physics, V Holešovičkách 2, 18000 Prague 8, Czech Republic

198 : Astronomical Observatory of Ivan Franko National University of Lviv, 8 Kyryla i Mephodia Street, Lviv, 79005, Ukraine

199 : Kobayashi-Maskawa Institute (KMI) for the Origin of Particles and the Universe, Nagoya University, Chikusa-ku, Nagoya

464-8602, Japan

200 : Graduate School of Technology, Industrial and Social Sciences, Tokushima University, Tokushima 770-8506, Japan

201 : Space Research Centre, Polish Academy of Sciences, ul. Bartycka 18A, 00-716 Warsaw, Poland

202 : Instituto de Física - Universidade de São Paulo, Rua do Matão Travessa R Nr.187 CEP 05508-090 Cidade Universitária,

São Paulo, Brazil

203 : International Institute of Physics at the Federal University of Rio Grande do Norte, Campus Universitário, Lagoa Nova CEP

59078-970 Rio Grande do Norte, Brazil

204 : University College Dublin, Belfield, Dublin 4, Ireland

205 : Centre for Astro-Particle Physics (CAPP) and Department of Physics, University of Johannesburg, PO Box 524, Auckland

Park 2006, South Africa

206 : Departamento de Física, Facultad de Ciencias Básicas, Universidad Metropolitana de Ciencias de la Educación, Santiago, Chile

207 : Núcleo de Formação de Professores - Universidade Federal de São Carlos, Rodovia Washington Luís, km 235 CEP

13565-905 - SP-310 São Carlos - São Paulo, Brazil

208 : Physik-Institut, Universität Zürich, Winterthurerstrasse 190, 8057 Zürich, Switzerland

209 : Department of Physical Sciences, Aoyama Gakuin University, Fuchinobe, Sagamihara, Kanagawa, 252-5258, Japan

210 : University of the Free State, Nelson Mandela Avenue, Bloemfontein, 9300, South Africa

211 : Faculty of Electronics and Information, Warsaw University of Technology, ul. Nowowiejska 15/19, 00-665 Warsaw, Poland

212: Rudjer Boskovic Institute, Bijenicka 54, 10000 Zagreb, Croatia

213 : Department of Physics, Konan University, Kobe, Hyogo, 658-8501, Japan

214 : Kumamoto University, 2-39-1 Kurokami, Kumamoto, 860-8555, Japan

215 : University School for Advanced Studies IUSS Pavia, Palazzo del Broletto, Piazza della Vittoria 15, 27100 Pavia, Italy

216: Aalto University, Otakaari 1, 00076 Aalto, Finland

217: Agenzia Spaziale Italiana (ASI), 00133 Roma, Italy

218: Observatoire de la Cote d'Azur, Boulevard de l'Observatoire CS34229, 06304 Nice Cedex 4, Franc 